Article

\title{
Environmental Policy Making in Supply Chains under Ambiguity and Competition: A Fuzzy Stackelberg Game Approach
}

\author{
Mina Rahimi ${ }^{1}$, Ashkan Hafezalkotob ${ }^{1}$, Sobhan Asian ${ }^{2, *(\mathbb{D})}$ and Luis Martínez ${ }^{3} \mathbb{D}$ \\ 1 College of Industrial Engineering, South Tehran Branch, Islamic Azad University, Tehran 1584743311, Iran; \\ minarahimi9@yahoo.com (M.R.); a_hafez@azad.ac.ir (A.H.) \\ 2 La Trobe Business School, La Trobe University, Melbourne, VIC 3086, Australia \\ 3 Department of Computer Science, University of Jaén, 23071 Jaén, Spain; martin@ujaen.es \\ * Correspondence: S.Asian@latrobe.edu.au
}

Citation: Rahimi, M.; Hafezalkotob,

A.; Asian, S.; Martínez, L.

Environmental Policy making in

Supply Chains under Ambiguity and

Competition: A Fuzzy Stackelberg

Game Approach. Sustainability 2021,

13, 2367. https://doi.org/

$10.3390 /$ su13042367

Academic Editors: Mabel C. Chou,

Shih-Che Lo and Prateek Jain

Received: 14 January 2021

Accepted: 6 February 2021

Published: 22 February 2021

Publisher's Note: MDPI stays neutral with regard to jurisdictional claims in published maps and institutional affiliations.

Copyright: (c) 2021 by the authors. Licensee MDPI, Basel, Switzerland. This article is an open access article distributed under the terms and conditions of the Creative Commons Attribution (CC BY) license (https:// creativecommons.org/licenses/by/ $4.0 /)$.

\begin{abstract}
Despite the substantial efforts of governments in promoting sustainable development, there exists considerable debate regarding the environmental policy making approach under information ambiguity and competition. This study investigates market competition between a green and a non-green supply chain (SC) under two government regulation policies, namely, selling price and production quantities. To tackle the policy making challenges, a fuzzy game theoretical model was employed in a centralized and decentralized SC setting. The results revealed that SCs always achieve a higher expected profit under a decentralized structure, regardless of the type of the governments intervention policy. Also, the government's policy making success was found to be highly dependent on the channel leadership, market competition, and the SC structure. Our findings suggest that the policy makers' objectives in reducing environmental pollution and increasing revenue are highly achievable, without risk of losing channel coordination and maximum level of efficiency.
\end{abstract}

Keywords: supply chain sustainability; environmental policy making; fuzzy Stackelberg game; sequential decision-making; information ambiguity

\section{Introduction}

Today, many academics and practitioners believe that environmental pollution is a significant problem, threatening the lives of people, animals, and plants. The amount of carbon dioxide in the air, which was about 280 units per million before the industrial revolution, had risen to 380 units per million in 2009, and this trend is accelerating [1]. One way to mitigate the risks and reverse the situation is to make appropriate social, technological, and political reforms in managing global supply chains (SCs) and move toward sustainable development goals [2,3]. Adopting green supply chain (GSCM) management concepts offers several benefits, such as saving energy resources, reducing carbon emissions, adding more value for customers, and enhancing company competitiveness $[4,5]$.

Some governments have recently recognized the urgency of emission reduction and proposed feasible carbon emission regulations [6]. To induce industries to produce greener products, governments employ systematic incentives and financially reward industries that produce less environmental pollution. On the other hand, environmental taxes can be used to achieve optimal allocation of resources by enforcing companies to produce and offer greener products and services [7]. Government intervention plays an important role in promoting green supply chains (GSCs) and is widely known as an instrumental factor in the successful implementation of environmental policies [8]. For example, the government of China has devised a comprehensive environmental impact taxation system to reduce pollution discharge. Such interventions create significant opportunities for the GSCs to mitigate environmental risks, while stimulating investments in green production. This eventually lead traditional SCs to move toward sustainable development goals [2,9]. In- 
spired by these facts, the present study is aimed at investigating the impacts of government intervention on pricing and quantity decisions in GSCs.

The business world has witnessed the rise of consumer awareness regarding the environmental aspects of products, and recognizes the growing concerns about increasing carbon emissions and limited waste disposal capacity. The ability to respond to incomplete information and ambiguity in the consumers' demand and manufacturing costs has also become one of the key success factors in competitive markets. Hence, the existence of complete information about consumer demand and manufacturing costs is no longer a valid assumption in the business environment [10]. The existing information ambiguity has caused considerable complexity and imposed substantial pressure on policy makers, who are already challenged by the rapidly evolving socio-economic problems [11]. To address these issues, both practitioners and academics have recently started to develop robust models that incorporate SC uncertainties and information ambiguity in environmental policy making problems [12].

There are various methods that can be used in policy making problems (e.g., quantitative decision-making, simulation-based methods, operation research). Most existing studies on policy making consider SCs and the government as independent decision-makers. Game theory provides a practical approach to examine and formulate the members' interactions in a multiplayer setting, where each player seeks to maximize its goals $[7,8]$. Due to the power asymmetry in the policy making problem, leader-follower Stackelberg game models have been widely used as effective tools. The government is commonly considered as the game leader, with the authority to move first and implement a variety of mechanisms in target industries. In line with the prior studies, our proposed methodology is constructed based on a Stackelberg game framework in which the lower level of the decision-making structure consists of two competing green and non-green SCs, while the government leads the game as the Stackelberg leader.

The present study focuses on the interaction between two competing green and regular SCs to investigate the impact of information ambiguity on the successful implementation of environmental policies. To this aim, some of the key SC parameters are considered as fuzzy numbers to incorporate information ambiguity into the policymaker's problem regarding the characteristics of competing SCs. This paper examines two frequently studied environmental policies in competitive markets: selling price and production quantity strategy. The following research questions will be addressed in this study:

1. How do the SC structure (centralized vs. decentralized) and information ambiguity affect the competing SCs' pricing decisions under different environmental policies?

2. How do government environmental policies affect the pricing decisions, the expected profit of centralized and decentralized SCs, and the environmental impact of SCs?

3. What are the impacts of environmental protection and revenue-seeking objectives of government on the overall performance of competing SCs under centralized and decentralized structures?

The remainder of this study is organized as follows: Section 2 provides a review of existing literature. Section 3 introduces the assumptions and mathematical notations. Section 4 describes the model formulation and presents four potential decision-making scenarios. Numerical results and discussion are provided in Section 5. Finally, we conclude remarks and make suggestions for future research in Section 6.

\section{Literature Review}

This study touches upon two important elements in the field of GSCM; namely, Stackelberg game/fuzzy game theory and government financial intervention. Each body of relevant literature is briefly reviewed in the following subsections, and then research gaps and the key contributions of this paper are presented. A summary of previous relevant quantitative studies is shown in Table 1. 


\subsection{Stackelberg Game and Fuzzy Game Theory in SC}

Stackelberg game models have been widely employed in applied economy and industry applications [13-15]. They deal with the leader(s)-follower(s) interaction in which the competition is not necessarily about a common goal, but players with different privileges compete to improve their own situation and increase their profits. Asian and Nie [16] used a sequential two-level optimization process as a solution approach for option contracts in a decentralized SC composed of one buyer, an unreliable main supplier, and a perfectly reliable backup supplier. They examined two benchmark and contract models under decentralized and centralized structures and analytically characterized the conditions under which the proposed option contract led to a win-win solution and coordinated the decentralized SC. Hadi et al. [17] formulated a game theoretical model in which the government applies some economic incentives to manage environmental effects under different scenarios. They considered two SCs, producing and offering non-green and green products in a shared market. The results indicated that collaboration between the recycler and retailer in the SC had a significant impact on the SC members' profit. Li and Chen [18] developed a Stackelberg game theoretical model in which the retailer sold customers a product under two different brands (quality standards), employing three strategies (i.e., selling two brands supplied by two competing manufacturers or integrating backward with either the high- or the low-quality manufacturer). Chen et al. [19] investigated the impact of the power relationship in both vertical and horizontal competition in a SC and analyzed the effects of customer demand in the bidimensional power dynamics of the SC. Yang et al. [20] used a Stackelberg game to analyze interactions between stations and roadside units that competed in an intelligent transportation system that provided information to drivers and passengers about traffic safety, traffic efficiency, autonomous driving, and entertainment.

Fuzzy game theory, with a discrete or continuous strategy domain, has been widely studied in the literature [21,22]. Cunlin and Qiang [23] investigated two-person zerosum games in the fuzzy environment using the Nash equilibrium strategy. Oliveira and Petraglia [24] proposed an application of the fuzzy optimization algorithm for finding the Nash equilibrium in formal games. Some studies in the literature focused on the factors which cannot be determined with certainty in real-world scenarios, such as the customer demand characteristics (e.g., quality, costs, and priority). For instance, Gumus and Guneri [25] considered nondeterministic parameters for demand, delivery times, and costs in a three-level SC. In addition, various methods of operations research, expert systems, multicriteria decision-making, control theory, and artificial intelligence have been developed to incorporate fuzzy parameters in SCs.

Zhao et al. [10] studied the fuzzy game approach to analyze the interactions between two substitutable products and used a Stackelberg game method to study the SC pricing decisions in fuzzy and uncertain environments. Similarly, Zhao and Wang [26] analyzed decisions about pricing and retail services in a SC in a fuzzy and uncertain environment. They examined the natural conflicts among the decision-makers in the construction sector. Feng et al. [27] examined the Stackelberg-Nash equilibrium and proposed a new model for evaluating manufacturing and construction material suppliers in a fuzzy environment. Wei and Zhao [28] considered product collection costs as fuzzy parameters, and used a game theory approach to find equilibrium decisions of the manufacturer, retailer, and a third party in a closed loop SC. Jamali et al. [29] investigated an SC pricing problem of a manufacturer and a retailer considering two distribution channels. A game theory approach was employed to obtain equilibrium solutions for cooperative and noncooperative scenarios. It was concluded that to boost the entire SC profit, an increase in the product greenness level was essential. Wang [30] characterized consumer demands, manufacturing costs, and warranty costs as fuzzy variables and evaluated the closed form optimal values of pricing and warranty decisions in a manufacturer-led SC Stackelberg setting. Zhao et al. [31] used game theory under symmetric and asymmetric information about retailer's product collection parameters in a two-echelon closed-loop SC. Fallah et al. [32] established Nash and Stackelberg game models to study competition in a closed loop SC in an uncertain 
environment. Yang and Xiao [8] used a game theory approach and studied the interaction between government and a GSC, considering fuzzy parameters for manufacturing costs and demand. Alamdar et al. [33] investigated the fuzzy price and sales effort/demand in a closed loop SC with a new decision-making structure. They also proposed a contract for coordinating a decentralized SC that was beneficial for all parties.

\subsection{Government Intervention}

In this section, we review the studies that focus on how governments impose environmental policies to encourage SCs to reduce environmental pollution. Tsireme et al. [34] explored the effects of managerial decisions to adopt new practices in GSCM in response to government environmental policies. Wang et al. [35] considered the relationships among governmental intervention, the provincial pollution-emission efficiency, and market development. The results showed that there was a positive correlation between market development and provincial pollution emission efficiency. Governmental intervention and market development were also found to be complementary factors in promoting China's provincial pollution-emission efficiency. Zhao et al. [10] used a game-theory model to analyze manufacturers' policies in GSCs, aiming to limit the environmental risk of materials and carbon emissions by focusing on tolerability of risk. They also showed that, by introducing a reasonable subsidy, a government can motivate producers to make appropriate restructuring decisions. Mahmoudi and Rasti-Barzoki [36] used a game theory approach under three different scenarios of government intervention in sustainable SCs. Their results showed that government policy affected the producers' activities, competitive markets, and carbon emissions. It was found that the imposed tariffs were the most effective government approach in minimizing environmental impacts. Chen et al. [37] considered a Stackelberg game between a manufacturer and a retailer in a dual channel closed loop SC under a government penalty-subsidy mechanism. The government rewarded or punished the manufacturer on the basis of the collection rate of the used products. Jin and Mei [38] established a game model between government and suppliers in a GSC under equilibrium conditions. They showed that governments can adjust their economic policy according to their supervision costs, the penalties on regular products, and the environmental damage costs of regular products. Yang and Xiao [8] studied the influence of government financial involvement in a GSC.

The effect of government environmental policies on the competitiveness of SCs is another relevant topic. Hafezalkotob $[39,40]$ investigated equilibrium solutions between green and regular products under different government intervention schemes in which the government was considered as a leader. They found that specific boundaries existed for government tariffs that guarantee a stable competitive market. $\mathrm{Wu}$ [41] considered a similar case in which the government intervened in a closed loop SC by imposing penalties or subsidies to reduce the environmental burden. After proposing six different policies, the results demonstrated that the proposed policies could effectively reduce the environmental burden and mitigate the intensity of price competition. Reza-Gharehbagh et al. [42] developed a model for two competing financial chains under governmental interventions. They analyzed the developed models by minimizing the arbitrages caused by speculative transactions to prohibit an excessively high interest rate.

Wang et al. [43] used a two-stage Stackelberg game model to develop an optimal scheme for a carbon emission tax. They analyzed how carbon emissions affect the selection of transportation modes and social welfare. Their study indicated that inflicting a greenhouse gas tax on a product at higher production costs, larger product volumes, or higher product density can increase the possibility of social welfare improvement. Similarly, Sheu and Chen [9] considered the government as a leader player in cooperative and noncooperative settings, and studied different green taxation strategies. Results indicated that a higher efficiency reduced the impact of financial interventions on the GSCs players' profits. Hafezalkotob et al. [44] considered the importance of government regulations and customer demands in managing the environmental issues. They found that such regulations may 
lead the organizations to review the critical measures that are required for the implementation of the GSCM while maintaining profits. Moradinasab et al. [45] modeled competition between government-led and private sector-led petroleum SCs by using Stackelberg and Nash game approaches. Hafezalkotob [46,47] developed price-energy-saving cooperation models for two GSCs competing under government intervention.

Table 1. A summary of previous relevant quantitative studies.

\begin{tabular}{|c|c|c|c|c|c|}
\hline \multirow{2}{*}{ Reference } & \multirow{2}{*}{ Fuzzy Variables } & \multicolumn{2}{|c|}{ Game Theory } & \multicolumn{2}{|c|}{ Intervention Policy } \\
\hline & & Stackelberg & Nash & Price & Others \\
\hline Yang et al. [8] & $\begin{array}{l}\text { Manufacturing cost; consumer demand; } \\
\text { market scale; green manufacturer's profit; } \\
\text { retailer's profit; green SC profit; retailer's profit; } \\
\text { price elasticity of the green product; green level } \\
\text { sensitivity coefficient of the product }\end{array}$ & $\sqrt{ }$ & $x$ & $\sqrt{ }$ & $x$ \\
\hline Sheu et al. [9] & $\times$ & $x$ & $\sqrt{ }$ & $\sqrt{ }$ & $\times$ \\
\hline Zhao et al. [10] & Consumer demand; manufacturing cost & $\times$ & $\sqrt{ }$ & $x$ & $x$ \\
\hline Wei et al. [11] & $\begin{array}{l}\text { Customer demand; remanufacturing cost; } \\
\text { the collecting cost }\end{array}$ & $\times$ & $\sqrt{ }$ & $\times$ & $\times$ \\
\hline Gao et al. [14] & $\times$ & $\sqrt{ }$ & $\times$ & $\times$ & $\times$ \\
\hline Hadi et al. [17] & $\times$ & $\sqrt{ }$ & $x$ & $\sqrt{ }$ & $x$ \\
\hline Cunlin et al. [23] & Players' profit & $\times$ & $\sqrt{ }$ & $\times$ & $\times$ \\
\hline Zhao et al. [26] & $\begin{array}{l}\text { Customer demands; manufacturing cost; service } \\
\text { cost coefficients }\end{array}$ & $\sqrt{ }$ & $\sqrt{ }$ & $\times$ & $\times$ \\
\hline Feng et al. [27] & $\begin{array}{l}\text { Production cost coefficient; inventory cost } \\
\text { coefficient; transportation cost coefficient; } \\
\text { transportation time coefficient; quantity of } \\
\text { materials; demand for materials }\end{array}$ & $\sqrt{ }$ & $\sqrt{ }$ & $x$ & $x$ \\
\hline Wei et al. [28] & $\begin{array}{l}\text { Demand; manufacturing cost; used products } \\
\text { collection costs }\end{array}$ & $\times$ & $\sqrt{ }$ & $x$ & $x$ \\
\hline Jamali et al. [29] & Wholesale; price, greening level of the product & $\times$ & $\sqrt{ }$ & $\times$ & $\times$ \\
\hline Wang [30] & $\begin{array}{l}\text { Consumer demand; manufacturing cost; } \\
\text { warranty cost }\end{array}$ & $\sqrt{ }$ & $\times$ & $\times$ & $\times$ \\
\hline Fallah et al. [32] & $\begin{array}{l}\text { Demand of SC; self-price and cross-price } \\
\text { elasticity coefficients }\end{array}$ & $\sqrt{ }$ & $\times$ & $\times$ & $x$ \\
\hline Alamdar et al. [33] & Price; sales effort-dependent demand & $\times$ & $\sqrt{ }$ & $\times$ & $\times$ \\
\hline Tsireme et al. [34] & $\times$ & $\sqrt{ }$ & $x$ & $\sqrt{ }$ & $x$ \\
\hline Mahmoudi et al. [36] & $\times$ & $\sqrt{ }$ & $\times$ & $\sqrt{ }$ & $\sqrt{ }$ \\
\hline Hafezalkotob [39] & $x$ & $\sqrt{ }$ & $x$ & $\sqrt{ }$ & $x$ \\
\hline Hafezalkotob [40] & $\times$ & $\sqrt{ }$ & $\times$ & $\sqrt{ }$ & $\sqrt{ }$ \\
\hline Moradinasab et al. [45] & $x$ & $\sqrt{ }$ & $\sqrt{ }$ & $\sqrt{ }$ & $\times$ \\
\hline Hafezalkotob [47] & $\times$ & $\sqrt{ }$ & $\times$ & $\sqrt{ }$ & $\sqrt{ }$ \\
\hline Liu et al. [48] & Manufacturing cost; demand & $\sqrt{ }$ & $\times$ & $x$ & $\times$ \\
\hline This paper & $\begin{array}{l}\text { Market demand for the retailers; costs; } \\
\text { manufacturers' profit }\end{array}$ & $\sqrt{ }$ & $\times$ & $\sqrt{ }$ & $\sqrt{ }$ \\
\hline
\end{tabular}

\subsection{Research Gaps and Key Contributions}

Due to the lack of accurate data and incomplete information, environmental policy goals are often set based on several assumptions and approximations. Traditionally, such ambiguities were simply ignored by the analysts and policy makers. With the rise of their awareness, some governments have started to consider information ambiguity more seriously by understanding, accepting, and managing different aspects of the problem.

The literature review confirmed that there is only a limited number of studies that incorporate information ambiguity in policy making problems. Some studies suggest that the existing ambiguities in environmental policy making can be captured and quantified through fuzzy estimation $[48,49]$. However, none of the existing studies simultaneously considered SC competition and information ambiguity, which are in fact the key characteristics of today's competitive business environment. To bridge the gap, we propose a 
Fuzzy game theoretical model between a GSC and an RSC (i.e., regular SC) and investigate government environmental intervention under competition and ambiguity. Using a bilevel multi-objective fuzzy optimization and considering both centralized and decentralized settings for two competing SCs, we investigate the impact of government environmental policies (i.e., selling price and production quantity) under different scenarios. A detailed sensitivity analysis and a numerical study are conducted to validate our theoretical results. Practical implications and managerial insights are also provided.

\section{Assumptions and Mathematical Notations}

We considered two competing green and regular (non-green) SCs. For simplicity, let GSC represent the SC that produces green products, and RSC denote the SC that produces regular products. Table 2 lists the mathematical notations that are used throughout this paper.

Table 2. A list of notations.

\begin{tabular}{ll}
\hline Notations & \\
\hline$\widetilde{a}_{i}$ & the market scale of product $i$ (fuzzy variable) \\
$\widetilde{c}_{i}$ & the marginal manufacturing cost of product $i$ (fuzzy variable) \\
$\widetilde{d}$ & the substitutability coefficient of product types $0 \leq \widetilde{d} \leq 1$ (fuzzy variable) \\
$p_{i}$ & the retailer price of product $i$ presented by retailer in SC type $i$ \\
$\theta_{i}$ & the environmental impact of product $i$ presented by SC type $i$ \\
$F$ & the minimum net revenue of the government \\
$E$ & the maximum environmental impact of products \\
$\widetilde{L}_{i}$ & the reservation profit of SC type $i$ (fuzzy variable) \\
$\widetilde{L}_{M i}$ & the reservation profit of manufacturer in SC type $i$ \\
$\widetilde{L}_{R i}$ & the reservation profit of retailer in SC type $i$ \\
$w_{i}$ & the unit wholesale price of product $i$ determined by the manufacturer in SC type $i$ \\
$t_{i}$ & the tariff for a unit of product $i$ set by the government \\
$G \widetilde{N} R$ & the government's net revenue obtained from tax, punishment, or incentive (fuzzy \\
$E \widetilde{I} S$ & variable) \\
$\widetilde{Q}$ & the total environmental impacts of the SCs' products (fuzzy variable) \\
$\widetilde{x_{i}}$ & the production quantity goal set by government for product $i$ \\
\hline
\end{tabular}

To avoid trivial cases and ensure the tractability of the mathematical models, the following assumptions were made:

(1) Following Hafezalkotob [39], we used environmental impact factors to represent and differentiate the pollution levels of green and regular products. This can be justified by the fact that, depending on the industry, different products cause different level of pollutions that can be measured by factors, such as a reversible index of products, reproduction or recycling index, and annual exhausting $\mathrm{CO}_{2}$ or $\mathrm{NO}_{\mathrm{x}}$ of products [47].

(2) In the real-world environment, the key parameters affecting policy making are usually vague and incomplete and, hence, cannot be estimated accurately. In this paper, information ambiguity is represented in the form of fuzzy numbers. Following Yang and Xiao [8], we considered some of our game parameters as trapezoidal fuzzy numbers.

(3) In all studied scenarios, the government was a Stackelberg leader who financially intervenes in the market with a tax or subsidy that indirectly influences the selling price of final products.

\section{Model Formulation and Analysis}

In this section, we first describe the research problem and analyze the basic concepts of the implemented game models. We then formulate the mathematic models and characterize the decision-making behavior of the SC members and government in different scenarios.

Consider two competing SCs, one manufacturer and one retailer, in a specific industry with one offering a green product and another a regular product in a shared market. These 
two products are substitutable with fuzzy variable $\widetilde{d}$ in the shared market. Following Bertrand's pricing competition model [50], customer demand is a function of the retailer's announced price, and the manufacturers must decide on the wholesale price. In general, SC decisions are made in either a centralized or a decentralized environment, referred to as the SC structure [16]. In a centralized structure, a single decision maker, with enough authority within the SC, makes decisions for the entire chain's members. In this case, the members are in line with the decision made by the centralized planner. However, since the SC members are often separate entities, they may be reluctant to follow decisions adopted for the whole SC and try to achieve their individual goals instead of optimizing the whole system. Therefore, the decentralized SC seems to be a more realistic model in most of the cases [51].

In this study, we consider both centralized and decentralized SC settings and investigate the competing SCs' decision-making when the government financially intervenes to control and reduce the environmental impact of the promoted products. Fuzzy decisionmaking can be generally divided into two groups: symmetric and asymmetric formulations. In a symmetric formulation, there is no significant difference between the weights of constraints and objective functions. However, in an asymmetric formulation, goals and constraints are not treated equally, and the weights applied have different meanings. In this paper, we follow Hafezalkotob [39] and Jiménez, Arenas, Bilbao, and Rodrı [52], and consider the fuzzy numbers rating based on their expectancy comparison.

From the government point of view, two main policies are often utilized to enhance the SC performance; namely, price-based and production quantity-based instruments. A price-based instrument is a market policy that can reduce or increase the market price of the products while contributing to the government's objectives. On the other hand, quantitybased instruments focus on the supply side of the SCs and are aimed to moderate the production quantity of products. Figure 1 depicts the different decision-making scenarios studied in this paper.

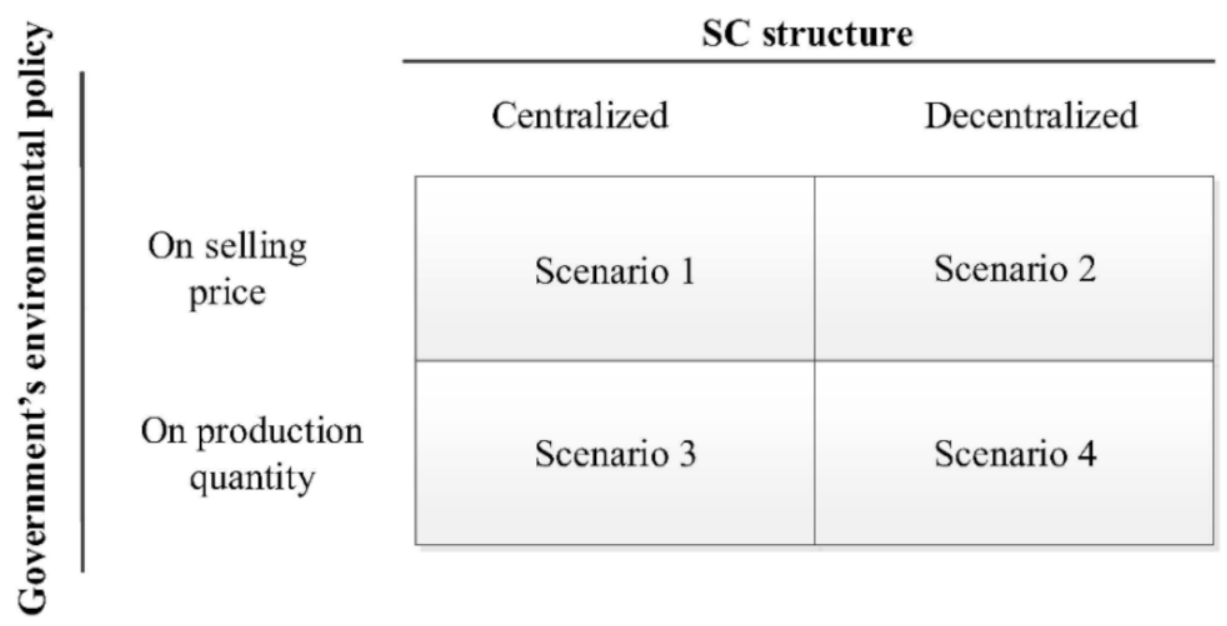

Figure 1. The different decision-making scenarios.

\subsection{Government Tariff on Selling Price under a Centralized SC (Scenario 1)}

Scenario 1 considers competition between two centralized SCs in which the government imposes its environmental policy on selling prices. The operational decisions made by manufacturers and retailers are often affected by the power of both vertical and horizontal members of the competitive game [19]. SCs consist of one manufacturer and one retailer playing a follower role, while the government is the leader. Thus, our main objective is to examine the effects of the government strategy by considering the payoff function for each follower (i.e., SC). Figure 2 illustrates the centralized structure of the SCs. 


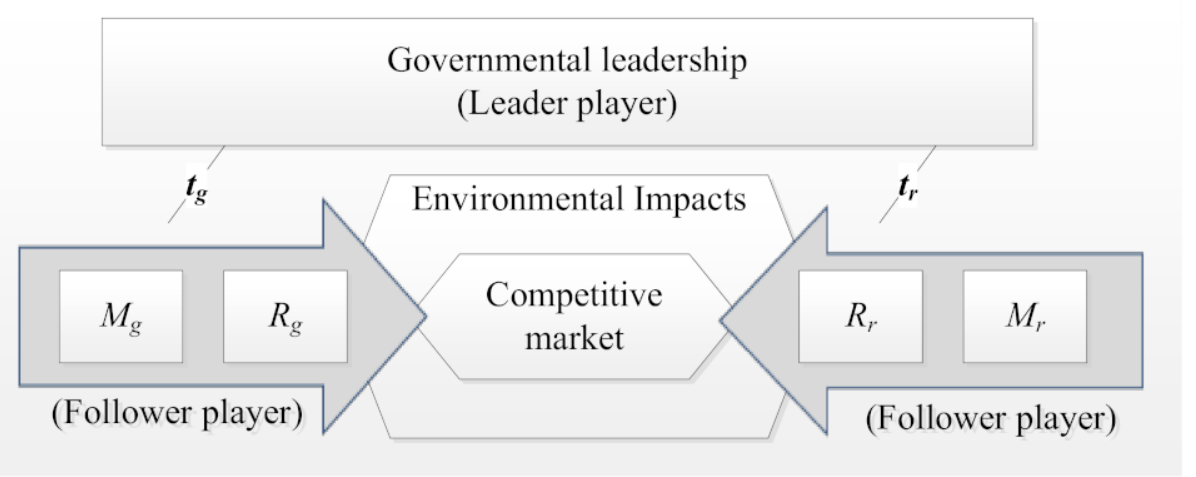

Figure 2. The schematic of SCs in a centralized structure under government tariffs.

Demand for product type $i(i=g, r)$ in both SCs can be defined as follows:

$$
\widetilde{q}_{i}\left(p_{i}, p_{j}, t_{i}, t_{j}\right)=\widetilde{a}_{i}-\left(p_{i}+t_{i}\right)+\widetilde{d}\left(p_{j}+t_{j}\right) ; i, j=g, r ; i \neq j
$$

We next derive the expected profit of the SCs as follows:

$$
E\left[\widetilde{\pi}_{i}\left(p_{i}, p_{j}, t_{i}, t_{j}\right)\right]=E\left[\left(p_{i}-\widetilde{c}_{i}\right)\left(\widetilde{a}_{i}-\left(p_{i}+t_{i}\right)+\widetilde{d}\left(p_{j}+t_{j}\right)\right)\right] ; i, j=g, r ; i \neq j
$$

Transforming Equation (2), we have:

$$
\begin{gathered}
E\left[\widetilde{\pi}_{i}\left(p_{i}, p_{j}, t_{i}, t_{j}\right)\right]= \\
p_{i} E\left[\widetilde{a}_{i}\right]+p_{i}\left(p_{j}+t_{j}\right) E[\widetilde{d}]-E\left[\widetilde{c}_{i} \widetilde{a}_{i}\right]+\left(p_{i}+t_{i}\right) E\left[\widetilde{c}_{i}\right]-\left(p_{j}+t_{j}\right) E\left[\widetilde{d} \widetilde{c}_{i}\right]-p_{i}\left(p_{i}+t_{i}\right)
\end{gathered}
$$

The expected profit in Equation (3) can be rewritten as:

$$
\begin{aligned}
E\left[\widetilde{\pi}_{i}\left(p_{i}, p_{j}, t_{i}, t_{j}\right)\right] & \\
& =1 / 2 \int_{0}^{1} p_{i}\left(\widetilde{a}_{i \alpha}^{L}+\widetilde{a}_{i \alpha}^{U}\right) \mathrm{d} \alpha \\
& +p_{i}\left(p_{i}+p_{j}\right) \int_{0}^{1}\left(\widetilde{d}_{i \alpha}^{L}+\widetilde{d}_{i \alpha}^{U}\right) \mathrm{d} \alpha \\
& +\left(p_{i}+t_{i}\right) \int_{0}^{1} 1 / 2\left(\widetilde{c}_{i \alpha}^{L}+\widetilde{c}_{i \alpha}^{U}\right) \mathrm{d} \alpha \int_{0}^{1} 1 / 2\left(\widetilde{c}_{i \alpha}^{U} \widetilde{a}_{i \alpha}^{L}+\widetilde{c}_{i \alpha}^{L} \widetilde{a}_{i \alpha}^{U}\right) \mathrm{d} \alpha \\
& -\left(p_{j}+t_{j}\right) \int_{0}^{1} 1 / 2\left(\widetilde{c}_{i \alpha}^{U} \widetilde{d}_{i \alpha}^{L}+\widetilde{c}_{i \alpha}^{L} \widetilde{d}_{i \alpha}^{U}\right) \mathrm{d} \alpha-p_{i}\left(p_{i}+t_{i}\right),
\end{aligned}
$$

where $\widetilde{a}_{\alpha}^{U}=\operatorname{Sup}\{r:\{\widetilde{C} \geq r\} \geq \alpha\}$ is the $\alpha$-optimistic and $\widetilde{a}_{\alpha}^{L}=\operatorname{Inf}\{r:\{\widetilde{C} \leq r\} \geq \alpha\}$ is the $\alpha$-pessimistic value of $\widetilde{\alpha}(0 \leq \alpha \leq 1)$. Similarly, this definition can be applied to other fuzzy parameters. Proposition 1 provides the optimal prices of products in Scenario 1.

Proposition 1. Under the centralized SC structure and when government tariffs are imposed on the selling price, the optimal price of SCs can be obtained as follows:

$$
p_{i}^{*}\left(t_{i}, t_{j}\right)=A_{i}+\frac{E[\widetilde{d}]^{2}-2}{4-E[\widetilde{d}]^{2}} t_{i}+\frac{E[\widetilde{d}]}{4-E[\widetilde{d}]^{2}} t_{j} ; i, j=g, r ; i \neq j
$$

where, $A_{i}=\frac{E\left[2 \widetilde{a}_{i}+2 \widetilde{c}_{j}+\widetilde{d} \widetilde{a}_{j}+\widetilde{d}_{c_{i}}\right]}{4-E\left[\widetilde{d}^{2}\right.}$.

All proofs are provided in the Appendix A. 
The government aims to maximize its net revenue (GNR) and environmental impacts (EIs) [39]. Therefore, the government's objectives function can be formulated as follows:

$$
\begin{aligned}
\operatorname{Max}_{x_{i}, x_{j}} E\left[G \widetilde{N} R\left(p_{i}, p_{j}, t_{i}, t_{j}\right)\right] & =\sum_{i \in\{r, g\}} t_{i} \widetilde{q}_{i}\left(p_{i}, p_{j}, t_{i}, t_{j}\right) \\
\operatorname{Min}_{x_{i}, x_{j}} E\left[E \widetilde{I} s\left(p_{i}, p_{j}, t_{i}, t_{j}\right)\right] & =\sum_{i \in\{r, g\}} \theta_{i} \widetilde{q}_{i}\left(p_{i}, p_{j}, t_{i}, t_{j}\right)
\end{aligned}
$$

s.t.

$$
\begin{gathered}
G \widetilde{N} R\left(p_{i}, p_{j}, t_{i}, t_{j}\right) \geq F \\
E \widetilde{I} s\left(p_{i}, p_{j}, t_{i}, t_{j}\right) \leq E \\
\widetilde{\pi}_{g}\left(p_{i}, p_{j}, t_{i}, t_{j}\right) \geq \widetilde{L}_{g} \\
\widetilde{\pi}_{r}\left(p_{i}, p_{j}, t_{i}, t_{j}\right) \geq \widetilde{L}_{r} \\
t_{i} \geq 0 ; i=r, g
\end{gathered}
$$

Constraints (8) and (9) ensure that GNR and EIs from both products are restricted to values $F$ and $E$, respectively. In addition, (10) and (11) are individual rational (IR) constraints in which minimum profits $\widetilde{L}_{g}$ and $\widetilde{L}_{r}$ are guaranteed for the green and regular SCs, respectively. The government model can be considered as a fuzzy multi-objective nonlinear problem (FMONLP) in that all parameters in objective functions and constraints are fuzzy. Therefore, for finding the government's tariffs, first of all, in Equations (6)(12), $p_{g}$ and $p_{r}$ can be replaced with the best response strategies, $p_{g}^{*}\left(t_{g}, t_{r}\right)$ and $p_{r}^{*}\left(t_{g}, t_{r}\right)$, provided in Proposition 1. Because of fuzzy constraints, the space solution of the model will also be a fuzzy set. Let $A^{U}$ and $A^{L}$ represent the upper and lower levels of a fuzzy variable $\widetilde{A}$, with $\alpha$-cut as the confidence level (i.e., $\widetilde{A}_{\alpha}=\left\{x \in X: \mu_{A}(x) \geq \alpha, \alpha \in[0,1]\right\}$ ). Considering an asymmetric fuzzy decision-making approach, the proposed fuzzy model (6)-(12) can be rewritten as follows:

$$
\begin{gathered}
\operatorname{Max} \zeta\left(t_{i}, t_{j}\right)=\operatorname{Max}\left\{\left(\omega_{k 1} \lambda_{k 1}\right)+\left(\omega_{k 2} \lambda_{k 2}\right)+\left(\omega_{k 3} \varphi_{k 3}\right)+\left(\omega_{k 4} \varphi_{k 4}\right)+\left(\omega_{k 5} \varphi_{k 5}\right)+\left(\omega_{k 6} \varphi_{k 6}\right)\right\} \\
\text { s.t. } \\
\lambda_{k 1} \leq \frac{G N R-G N R_{\alpha}^{L}}{G N R_{\alpha}^{U}-G N R_{\alpha}^{L}} \\
\lambda_{k 2} \leq \frac{E I s_{\alpha}^{U}-E I s}{E I s_{\alpha}^{U}-E I s_{\alpha}^{L}} \\
\varphi_{k 3} \leq \frac{F+G N R_{\alpha}^{U}}{G N R_{\alpha}^{U}-G N R_{\alpha}^{L}} \\
\varphi_{k 4} \leq \frac{E-E I s_{\alpha}^{L}}{E I s_{\alpha}^{U}-E I s_{\alpha}^{L}} \\
\varphi_{k 5} \leq \frac{\widetilde{\pi}_{g}\left(p_{i}, p_{j}, t_{i}, t_{j}\right)-\widetilde{L}_{g}^{L}}{\widetilde{L}_{g}^{U}-\widetilde{L}_{g}^{L}} \\
\varphi_{k 6} \leq \frac{\widetilde{\pi}_{r}\left(p_{i}, p_{j}, t_{i}, t_{j}\right)-\widetilde{L}_{r}^{L}}{\widetilde{L}_{r}^{U}-\widetilde{L}_{r}^{L}} \\
\lambda_{k 1}+\lambda_{k 2}+\varphi_{k 3}+\varphi_{k 4}+\varphi_{k 5}+\varphi_{k 6}=1 \\
\lambda_{k 1} \geq \alpha, \lambda_{k 2} \geq \alpha, \varphi_{k 3} \geq \alpha, \varphi_{k 4} \geq \alpha, \varphi_{k 5} \geq \alpha, \varphi_{k 6} \geq \alpha, \alpha \in[0,1] \\
0 \leq \lambda_{k 1}, \lambda_{k 2}, \varphi_{k 3}, \varphi_{k 4}, \varphi_{k 5}, \varphi_{k 6} \leq 1 \\
t_{i} \geq 0 ; i=r, g
\end{gathered}
$$


where $\lambda_{k 1}$ and $\lambda_{k 2}$ are the level access of the government's objective functions (6) - (7), and $\varphi_{k 3}, \varphi_{k 4}, \varphi_{k 5}$ and $\varphi_{k 6}$ refer to the level access of the fuzzy constraints (8)-(11). GNR $R_{\alpha}^{L}$, $G N R_{\alpha}^{U}, E I s_{\alpha}^{L}$, and $E I s_{\alpha}^{U}$ are lower bounds and upper bounds of GNR and EIs, respectively. The above single objective nonlinear programming model can be solved using an available nonlinear programming solver package, such as GAMS or Lingo.

\subsection{Government Tariff on Selling Price under a Decentralized SC (Scenario 2)}

In this scenario, the manufacturer and the retailer make their decisions independently in a decentralized setting. So, the power structure of SC channels is different from the previous scenario. The model setting is graphically illustrated in Figure 3.

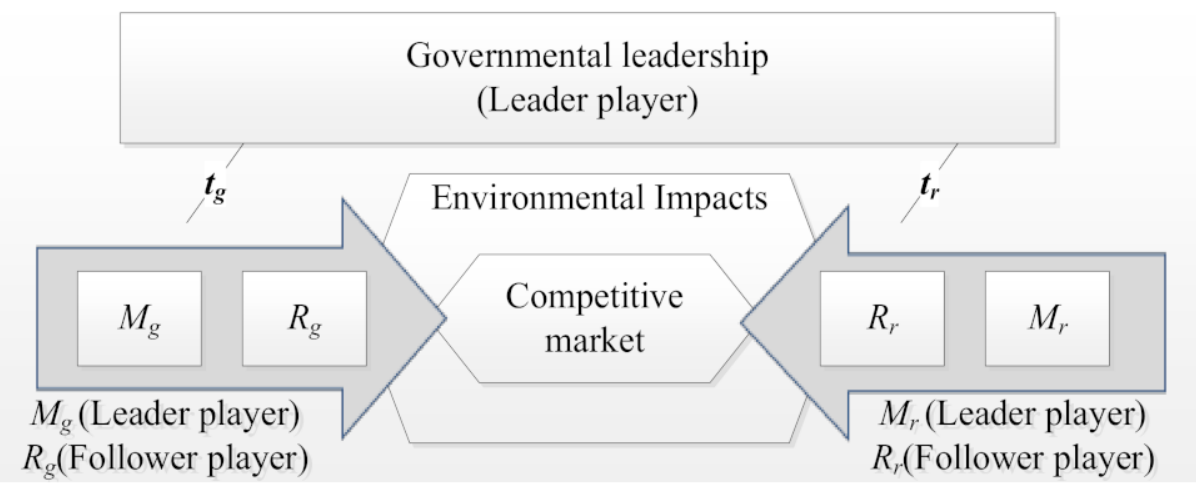

Figure 3. The schematic of SCs in a decentralized structure with government tariffs.

For $i=r, g$, there are three players in the game, including the government (the game leader), a manufacturer $M_{i}$ (the game subleader), and a retailer $R_{i}$ (the game follower). Government tariffs are imposed on retail prices of SCs decided by the retailers.

Following the backward induction procedure, we first focus on the followers' problem. Using the previously derived demand function (Equation (1)), the expected profit of the manufacturer and retailer (for $i, j=g, r ; i \neq j$ ) can be derived using Equations (24) and (25), respectively.

$$
\begin{aligned}
& \left.E\left[\tilde{\pi}_{R i}\left(p_{i}, p_{j}, t_{i}, t_{j}, \quad w_{i}, w_{j}\right)\right]\right]=E\left[\left(p_{i}-w_{i}\right) \widetilde{q}_{i}\left(p_{i}, p_{j}, t_{i}, t_{j}\right)\right] \\
& =\frac{p_{i}-w_{i}}{2} \int_{0}^{1}\left(\widetilde{a}_{i \alpha}^{U}+\widetilde{a}_{i \alpha}^{L}\right) \mathrm{d} \alpha \\
& +\frac{\left(p_{j}+t_{j}\right)\left(p_{i}-w_{i}\right)}{2} \int_{0}^{1}\left(\widetilde{d}_{\alpha}^{U}+\widetilde{d}_{\alpha}^{L}\right) \mathrm{d} \alpha-\left(p_{i}+t_{i}\right)\left(p_{i}-w_{i}\right) \\
& \left.E\left[\widetilde{\pi}_{M i}\left(p_{i}, p_{j}, t_{i}, t_{j}, w_{i}, w_{j}\right)\right]\right]=E\left[\left(w_{i}-\widetilde{c}_{i}\right) \widetilde{q}_{i}\left(p_{i}, p_{j}, t_{i}, t_{j}\right)\right]=w_{i} \int_{0}^{1} 1 / 2\left(\widetilde{a}_{i \alpha}^{U}+\widetilde{a}_{i \alpha}^{L}\right) \mathrm{d} \alpha+ \\
& \frac{w_{i}\left(p_{j}+t_{j}\right)}{2} \int_{0}^{1}\left(\widetilde{d}_{\alpha}^{U}+\widetilde{d}_{\alpha}^{L}\right) \mathrm{d} \alpha+\left(p_{i}+t_{i}\right) \int_{0}^{1}\left(\widetilde{c}_{i \alpha}^{U}+\widetilde{c}_{i \alpha}^{L}\right) \mathrm{d} \alpha-\left(p_{j}+t_{j}\right) \int_{0}^{1}\left(\widetilde{d}_{\alpha}^{L} \widetilde{c}_{i \alpha}^{U}+\widetilde{d}_{\alpha}^{U} \widetilde{c}_{i \alpha}^{L}\right) \mathrm{d} \alpha- \\
& \int_{0}^{1}\left(a_{i \alpha}^{L} \widetilde{c}_{i \alpha}^{U}+\widetilde{a}_{i \alpha}^{U} \widetilde{c}_{i \alpha}^{L}\right) \mathrm{d} \alpha-w_{i}\left(p_{i}+t_{i}\right)
\end{aligned}
$$

Proposition 2. Under the decentralized SC structure and when government tariffs are imposed on retail price, the optimal retail price of each SC can be obtained as follows:

$$
p_{i}^{*}\left(t_{i}, t_{j}, w_{i}, w_{j}\right)=B_{i}+\frac{2}{4-E[\tilde{d}]^{2}}\left(w_{i}\right)+\frac{E[\tilde{d}]}{4-E[\tilde{d}]^{2}}\left(w_{j}\right)
$$

where, $B_{i}=\frac{E\left[2 \widetilde{a}_{i}+\widetilde{d}_{\tilde{a}}\right]-\left(E[\widetilde{d}]^{2}-2\right) t_{i}+E[\widetilde{d}] t_{j}}{4-E[\widetilde{d}]^{2}} . i, j=g, r ; i \neq j$ 
Using $p_{i}^{*}\left(t_{i}, t_{j} w_{i}, w_{j}\right)$, the manufacturer's profit functions can be updated, and their optimal wholesale price is provided in Proposition 3.

Proposition 3. Under the decentralized SC structure and when government tariffs are imposed on retail price, the manufacturer's optimal wholesale price can be obtained as follows:

$$
w_{i}^{*}\left(t_{i}, t_{j}\right)=C_{i}+C_{1} t_{i}+C_{2} t_{j} ; \quad i, j=g, r ; \quad i \neq j
$$

where,

$$
\begin{aligned}
& C_{i}=\left(E\left[\left(2 \widetilde{d}^{4}-8 \widetilde{d}^{2}+8\right) \widetilde{c}_{i}\right]-E\left[\left(\widetilde{d}^{3}-2 \widetilde{d}\right) \widetilde{c}_{j}\right]-E\left[\left(-2 \widetilde{d}^{3}+6 \widetilde{d}\right) \widetilde{a}_{j}\right]+E\left[\left(-3 \widetilde{d}^{2}+8\right) \widetilde{a}_{i}\right]\right) / \\
& \left(E\left[4 \widetilde{d}^{4}-17 \widetilde{d}^{2}+16\right]\right) \\
& C_{1}=E\left[-5 \widetilde{d}^{4}-2 \widetilde{d}^{3}+25 \widetilde{d}^{2}+4 \widetilde{d}-24\right] / E\left[4 \widetilde{d}^{4}-17 \widetilde{d}^{2}+16\right] \text { and } \\
& C_{2}=E\left[2 \widetilde{d}^{5}+2 \widetilde{d}^{4}-2 \widetilde{d}^{3}+14 \widetilde{d}\right] / E\left[4 \widetilde{d}^{4}-17 \widetilde{d}^{2}+16\right]
\end{aligned}
$$

Replacing $w_{i}^{*}\left(t_{i}, t_{j}\right)$ in the government's objective functions (28)-(38), the model can be formulated as follows:

$$
\begin{aligned}
& \operatorname{Max}_{t_{i}, t_{j}} E\left[G \widetilde{N} R\left(p_{i}, p_{j}, t_{i}, t_{j}, w_{i}, w_{j}\right)\right]=E\left[\sum_{i \in\{g, r\}} t_{i} \widetilde{q}_{i}\left(p_{i}, p_{j}, t_{i}, t_{j}\right)\right] \\
& \operatorname{Min}_{t_{i}, t_{j}} E\left[E \widetilde{I} s\left(p_{i}, p_{j}, t_{i}, t_{j}, w_{i}, w_{j}\right)\right]=E\left[\sum_{i \in\{g, r\}} \theta_{i} \widetilde{q}_{i}\left(p_{i}, p_{j}, t_{i}, t_{j}\right)\right]
\end{aligned}
$$

s.t.

$$
\begin{gathered}
G \widetilde{N} R\left(p_{i}, p_{j}, t_{i}, t_{j}, w_{i}, w_{j}\right) \geq F \\
E \widetilde{I} s\left(p_{i}, p_{j}, t_{i}, t_{j}, w_{i}, w_{j}\right) \leq E \\
\widetilde{\pi}_{g}\left(p_{i}, p_{j}, t_{i}, t_{j}, w_{i}, w_{j}\right) \geq \widetilde{L}_{g} \\
\widetilde{\pi}_{r}\left(p_{i}, p_{j}, t_{i}, t_{j}, w_{i}, w_{j}\right) \geq \widetilde{L}_{r} \\
\widetilde{\pi}_{M g}\left(p_{i}, p_{j}, t_{i}, t_{j}, w_{i}, w_{j}\right) \geq L_{M g} \\
\widetilde{\pi}_{M r}\left(p_{i}, p_{j}, t_{i}, t_{j}, w_{i}, w_{j}\right) \geq L_{M r} \\
\widetilde{\pi}_{R g}\left(p_{i}, p_{j}, t_{i}, t_{j}, w_{i}, w_{j}\right) \geq L_{R g} \\
\widetilde{\pi}_{R r}\left(p_{i}, p_{j}, t_{i}, t_{j}, w_{i}, w_{j}\right) \geq L_{R r} \\
t_{i} \geq 0 ; i=r, g
\end{gathered}
$$

As in Scenario 1, we assume that the government's objectives are maximizing the expected revenue and minimizing the environmental impacts of SCs. Solving the model, the best solutions for government tariffs can be obtained. The game-theoretical model in (28)-(38) can be converted to a single objective model, and after using $\alpha$-cut, $w_{g}^{*}$ and $w_{r}^{*}$ can be obtained. For the sake of brevity, details of the solution process are omitted.

\subsection{Government Tariff on Production Quantity under a Centralized SC (Scenario 3)}

In this scenario, all SC decisions are made under a centralized structure, and the environmental tariff is imposed on the production quantity. The government is the game leader and determines a production quantity threshold $\left(Q_{g}\right.$ and $\left.Q_{r}\right)$, above which the SCs receive a government subsidy $\left(x_{g}, x_{r}\right.$ with a positive value). Otherwise, the $\mathrm{SCs}$ will incur a penalty fee $\left(x_{g}, x_{r}\right.$ with a negative value). In response to the government's decision, the centralized SCs (i.e., the followers) choose their retail price $\left(p_{g}^{*}\right.$ and $\left.p_{r}^{*}\right)$. Figure 4 illustrates the schematic of decision-making under this scenario. 


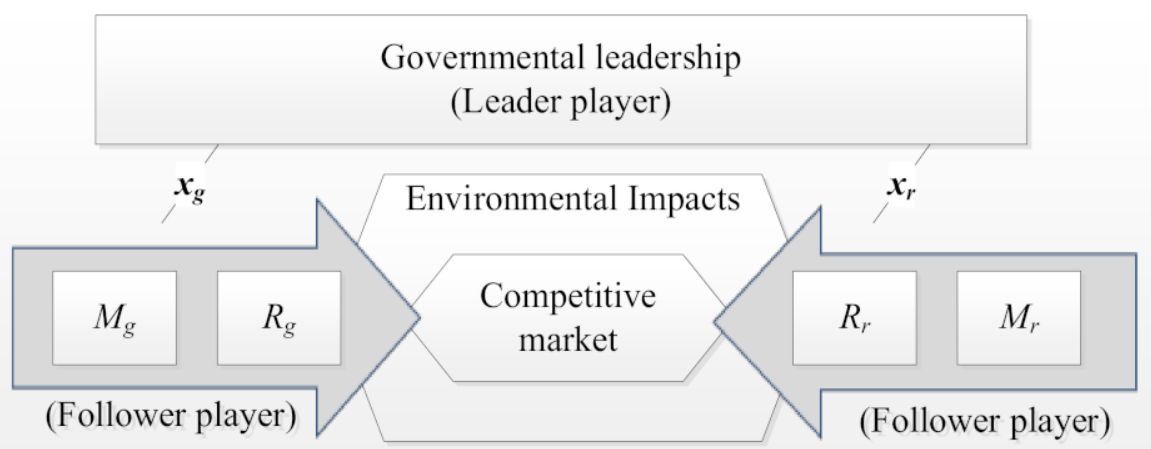

Figure 4. Centralized SC competition under government policy on production quantities. follows:

Under Scenario 3, the market demand for product type $i(i=g, r)$ can be defined as

$$
\widetilde{q}_{i}\left(p_{i}, p_{j}\right)=\widetilde{a}_{i}-p_{i}+\widetilde{d} p_{j} ; i, j=g, r ; i \neq j
$$

The expected profit of centralized SCs can be derived as follows:

$$
\begin{gathered}
E\left[\widetilde{\pi}_{g}\left(p_{i}, p_{j}, x_{i}, x_{j}\right)\right]=E\left[\left(p_{g}-\widetilde{c}_{g}\right) \widetilde{q}_{g}\left(p_{g}, p_{r}\right)+x_{g}\left(\widetilde{q}_{g}\left(p_{g}, p_{r}\right)-Q_{g}\right)\right] \\
E\left[\widetilde{\pi}_{r}\left(p_{i}, p_{j}, x_{i}, x_{j}\right)\right]=E\left[\left(p_{r}-\widetilde{c}_{r}\right) \widetilde{q}_{r}\left(p_{g}, p_{r}\right)-x_{r}\left(\widetilde{q}_{r}\left(p_{g}, p_{r}\right)-Q_{r}\right)\right]
\end{gathered}
$$

Using $\alpha$-optimistic and $\alpha$-pessimistic, the expected profit of GSC and RSC can be derived as follows:

$$
\begin{gathered}
2 E\left[\tilde{\pi}_{g}\left(p_{i}, p_{j}, x_{i}, x_{j}\right)\right]=\left(p_{g}+x_{g}\right) \int\left(a_{g \alpha}^{L}+a_{g \alpha}^{U}\right) \mathrm{d} \alpha+p_{r}\left(x_{g}-p_{g}\right) \int\left(d_{\alpha}^{L}+d_{\alpha}^{U}\right) \mathrm{d} \alpha \\
\quad-p_{r} \int\left(d_{\alpha}^{U} c_{g \alpha}^{L}+d_{\alpha}^{L} c_{g \alpha}^{U}\right) \mathrm{d} \alpha-\int\left(a_{\alpha}^{U} c_{g \alpha}^{L}+a_{\alpha}^{L} c_{g \alpha}^{U}\right) \mathrm{d} \alpha+p_{g} \int\left(c_{g \alpha}^{L}+c_{g \alpha}^{U}\right) \mathrm{d} \alpha \\
-2 p_{g}^{2}-2 x_{g} Q_{g}-2 x_{g} p_{g} \\
2 E\left[\tilde{\pi}_{r}\left(p_{i}, p_{j}, x_{i}, x_{j}\right)\right]=\left(p_{r}+x_{r}\right) \int\left(a_{r \alpha}^{L}+a_{r \alpha}^{U}\right) \mathrm{d} \alpha+p_{g}\left(p_{r}-x_{r}\right) \int\left(d_{\alpha}^{L}+d_{\alpha}^{U}\right) \mathrm{d} \alpha+ \\
2 x_{r} Q_{r}-\int\left(a_{\alpha r}^{U} c_{r \alpha}^{L}+a_{r \alpha}^{L} c_{r \alpha}^{U}\right) \mathrm{d} \alpha+p_{r} \int\left(c_{r \alpha}^{L}+c_{r \alpha}^{U}\right) \mathrm{d} \alpha-p_{g} \int\left(d_{\alpha}^{U} c_{r \alpha}^{L}+d_{\alpha}^{L} c_{r \alpha}^{U}\right) \mathrm{d} \alpha-2 p_{r}^{2}+ \\
2 x_{r} p_{r}
\end{gathered}
$$

Proposition 4. Under the centralized SC structure and when government tariffs are imposed on the production quantity of SCs, the manufacturer's optimal wholesale price can be obtained as follows:

$$
\begin{aligned}
& p_{g}^{*}\left(x_{i}, x_{j}\right)=D_{1 g}-\frac{2}{4-E[\tilde{d}]^{2}} x_{g}+\frac{E[\tilde{d}]}{4-E[\tilde{d}]^{2}} x_{r} \\
& p_{r}^{*}\left(x_{i}, x_{j}\right)=D_{2 r}-\frac{E[\tilde{d}]}{4-E[\tilde{d}]^{2}} x_{g}+\frac{2}{4-E[\tilde{d}]^{2}} x_{r}
\end{aligned}
$$

where, $D_{1 g}=\frac{2 E\left[\widetilde{a}_{g}+\widetilde{c}_{g}\right]+E\left[\widetilde{a}_{r} \widetilde{d}+\widetilde{c}_{r} \widetilde{d}\right]}{4-E[\widetilde{d}]^{2}}$ and $D_{2 r}=\frac{2 E\left[\widetilde{a}_{r}+\widetilde{c}_{r}\right]+E\left[\widetilde{a}_{g} \widetilde{d}+\widetilde{c}_{g} \widetilde{d}\right]}{4-E[\widetilde{d}]^{2}}$

Similar to Scenario 1, the government's objectives maximize the GNR and minimize the EIs.

$$
\operatorname{Max}_{x_{i}, x_{j}} E\left[G \tilde{N} R\left(p_{i}, p_{j}, x_{i}, x_{j}\right)\right]=E\left[\sum_{i \in\{g, r\}} x_{i} \widetilde{q}_{i}\left(p_{i}, p_{j}, x_{i}, x_{j}\right)\right]
$$




$$
\operatorname{Min}_{x_{i}, x_{j}} E\left[E \widetilde{I} s\left(p_{i}, p_{j}, x_{i}, x_{j}\right)\right]=E\left[\sum_{i \in\{g, r\}} \theta_{i} \widetilde{q}_{i}\left(p_{i}, p_{j}, x_{i}, x_{j}\right)\right]
$$

s.t.

$$
\begin{gathered}
G \widetilde{N} R\left(p_{i}, p_{j}, x_{i}, x_{j}\right) \geq F \\
E \widetilde{I} s\left(p_{i}, p_{j}, x_{i}, x_{j}\right) \leq E \\
\widetilde{\pi}_{g}\left(p_{i}, p_{j}, x_{i}, x_{j}\right) \geq \widetilde{L}_{g} \\
\widetilde{\pi}_{r}\left(p_{i}, p_{j}, x_{i}, x_{j}\right) \geq \widetilde{L}_{r} \\
x_{g}, x_{r} \geq 0
\end{gathered}
$$

Substituting $p_{g}^{*}\left(x_{i}, x_{j}\right)$ and $p_{r}^{*}\left(x_{i}, x_{j}\right)$, which were presented in Proposition 4 , into the above model (46)-(52), the government's optimal decision variables can be obtained.

\subsection{Government Tariff on Production Quantity under a Decentralized SC (Scenario 4)}

In this scenario, SC decisions are made independently under a decentralized structure, and the environmental policy of the government is applied to the production quantity of the SCs. Figure 5 depicts a three-player game model (among the government, manufacturers, and retailers) with a hierarchical power structure.

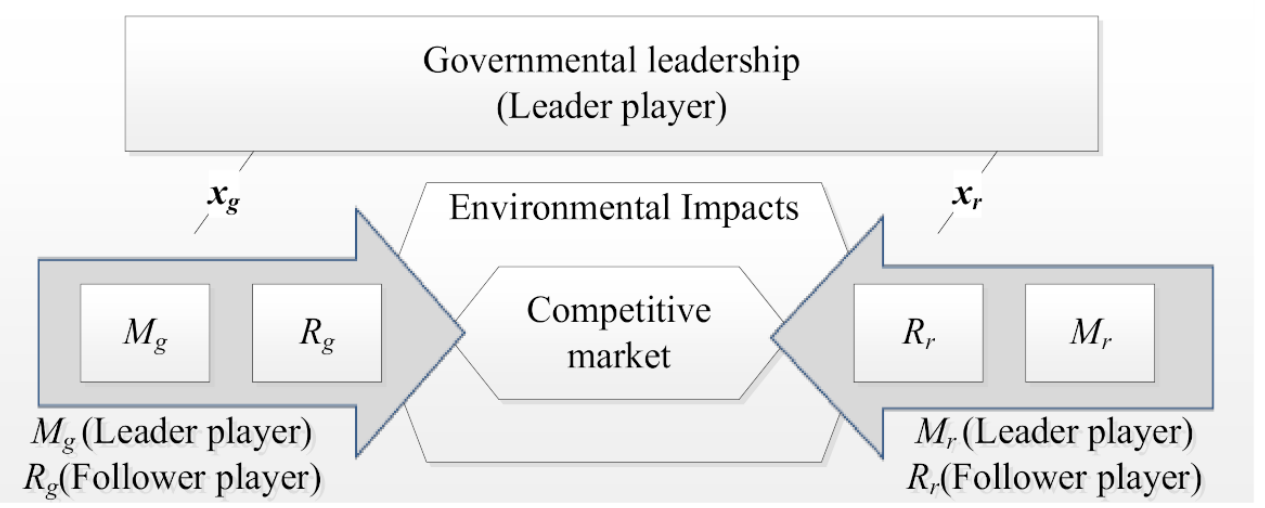

Figure 5. The schematic of SCs in a decentralized structure under governmental tariffs.

In response to the government's policies (i.e., $x_{g}$ and $x_{r}$ which can be positive or negative values), the expected profit of retailers and manufacturers (green and regular) in SCs are derived in Equations (53)-(55):

$$
\begin{gathered}
E\left[\widetilde{\pi}_{R i}\left(p_{i}, p_{j}, x_{i}, x_{j}, w_{i}, w_{j}\right)\right]=E\left[\left(p_{i}-w_{i}\right) \widetilde{q}_{i}\right]=\frac{p_{i}-w_{i}}{2} \int\left(a_{i \alpha}^{L}+a_{i \alpha}^{U}\right) \mathrm{d} \alpha+ \\
\frac{p_{j}\left(p_{i}-w_{i}\right)}{2} \int\left(d_{\alpha}^{L}+d_{\alpha}^{U}\right) \mathrm{d} \alpha-p_{i}\left(p_{i}-w_{i}\right) ; i, j=g, r ; i \neq j
\end{gathered}
$$

The expected profit of green and regular manufactures can be derived as follows:

$$
\begin{gathered}
E\left[\widetilde{\pi}_{M g}\left(p_{g}, p_{r}, x_{g}, x_{r}, w_{g}, w_{r}\right)\right]=\frac{x_{g}+w_{g}}{2} \\
\int\left(a_{g \alpha}^{L}+a_{g \alpha}^{U}\right) \mathrm{d} \alpha+\frac{p_{r}\left(x_{g}+w_{g}\right)+p_{g}+x_{r}}{2} \int\left(d_{\alpha}^{L}+d_{\alpha}^{U}\right) \mathrm{d} \alpha+ \\
\frac{p_{g}}{2} \int\left(c_{g \alpha}^{L}+c_{g \alpha}^{U}\right) \mathrm{d} \alpha-\frac{1}{2} \int\left(a_{\alpha g}^{U} c_{g \alpha}^{L}+a_{g \alpha}^{L} c_{g \alpha}^{U}\right) \mathrm{d} \alpha-p_{r} \\
\int\left(d_{\alpha}^{U} c_{g \alpha}^{L}+d_{\alpha}^{L} c_{g \alpha}^{U}\right) \mathrm{d} \alpha-w_{g} p_{g}-x_{g}\left(p_{g}-Q_{g}\right)
\end{gathered}
$$




$$
\begin{gathered}
E\left[\tilde{\pi}_{M r}\left(p_{g}, p_{r}, x_{g}, x_{r}, w_{g}, w_{r}\right)\right]=\frac{w_{r}-x_{r}}{2} \int\left(a_{r \alpha}^{L}+a_{r \alpha}^{U}\right) \mathrm{d} \alpha+\frac{p_{g} w_{r}}{2} \int\left(d_{\alpha}^{L}+d_{\alpha}^{U}\right) \mathrm{d} \alpha-Q_{r} x_{r}- \\
p_{r}\left(w_{r}+x_{r}\right)-\frac{1}{2} \int\left(a_{\alpha r}^{U} c_{r \alpha}^{L}+a_{r \alpha}^{L} c_{r \alpha}^{U}\right) \mathrm{d} \alpha+p_{r} \int \frac{1}{2}\left(c_{r \alpha}^{L}+c_{r \alpha}^{U}\right) \mathrm{d} \alpha-p_{g} \int \frac{1}{2}\left(d_{\alpha}^{U} c_{r \alpha}^{L}+\right. \\
\left.d_{\alpha}^{L} c_{r \alpha}^{U}\right) \mathrm{d} \alpha
\end{gathered}
$$

Proposition 5. Under the decentralized SC structure and when government tariffs are imposed on production quantity of SCs, the optimal retail price of each SC can be derived as follows:

$$
p_{i}^{*}\left(x_{i}, x_{j}, w_{i}, w_{j}\right)=H_{i}+\frac{2}{1-E\left[4 \widetilde{d}^{2}\right]} w_{i}+\frac{E[\tilde{d}]}{4-E[\widetilde{d}]^{2}} w_{j} ; i, j=g, r ; i \neq j
$$

where

$$
H_{i}=\frac{E\left[2 \widetilde{a}_{i}+\widetilde{d}_{j}\right]}{1-E\left[4 \widetilde{d}^{2}\right]} .
$$

After obtaining $p_{i}^{*}\left(t_{i}, t_{j}, w_{i}, w_{j}\right)$ for each SC, the manufacturers' optimal wholesale prices are computed by Proposition 6.

Proposition 6. Under the decentralized SC structure and when government tariffs are imposed on the production quantity of SCs, the manufacturer's optimal wholesale price can be obtained as follows:

$$
\begin{gathered}
w_{g}^{*}\left(x_{g}, x_{r}\right)=\frac{E\left[\widetilde{d}^{4}\right]\left(2 x_{g}+4\right)+E\left[\widetilde{d}^{3}\right]\left(2 E\left[\widetilde{a}_{r}\right]+4 E\left[\widetilde{c}_{g}\right]+E\left[\widetilde{c}_{r}\right]+4\right)+E\left[\widetilde{d}^{2}\right]\left(3 E\left[\widetilde{a}_{g}\right]+4 E\left[\widetilde{c}_{g}\right]-8 x_{g}-8\right)}{4 E\left[\widetilde{d}^{4}\right]-17 E\left[\widetilde{d}^{2}\right]+16}+ \\
\frac{-E[\widetilde{d}]\left(6 E\left[\widetilde{a}_{r}\right]+8 E\left[\widetilde{c}_{g}\right]+2 E\left[\widetilde{c}_{r}\right]-2 x_{r}+8\right)+8\left(x_{g}-E\left[\widetilde{a}_{g}\right]-E\left[\widetilde{c}_{g}\right]\right)}{4 E\left[\widetilde{d}^{4}\right]-17 E\left[\widetilde{d}^{2}\right]+16} . \\
w_{r}^{*}\left(x_{g}, x_{r}\right)=\frac{2 E\left[\widetilde{d}^{4}\right] E\left[\widetilde{c}_{r}\right]+E\left[\widetilde{d}^{3}\right]\left(-3 E\left[\widetilde{a}_{r}\right]+2 E\left[\widetilde{c}_{g}\right]-8 E\left[\widetilde{c}_{r}\right]+2\right)+E\left[\widetilde{d}^{2}\right]\left(2-3 E\left[\widetilde{a}_{r}\right]+2 E\left[\widetilde{c}_{g}\right]-8 E\left[\widetilde{c}_{r}\right]+8 x_{r}\right)}{4 E\left[\widetilde{d}^{4}\right]-17 E\left[\widetilde{d}^{2}\right]+16}+ \\
\frac{E[\widetilde{d}]\left(6 E\left[\widetilde{a}_{g}\right]+2 E\left[\widetilde{c}_{g}\right]-2 x_{g}\right)+8\left(-x_{r}+E\left[\widetilde{a}_{r}\right]+E\left[\widetilde{c}_{r}\right]\right)}{4 E\left[\widetilde{d}^{4}\right]-17 E\left[\widetilde{d}^{2}\right]+16}
\end{gathered}
$$

After obtaining the retailers' and the manufacturers' best response solutions, the government's model can be formulated as follows:

$$
\begin{gathered}
\operatorname{Max}_{x_{i}, x_{j}} E\left[G \tilde{N} R\left(p_{i}, p_{j}, x_{i}, x_{j}\right)\right]=E\left[\sum_{i, j=g, r} x_{i} \widetilde{q}_{i}\left(p_{i}, p_{j}\right)\right] \\
\operatorname{Min}_{x_{i}, x_{j}} E\left[E \widetilde{I} s\left(p_{i}, p_{j}, x_{i}, x_{j}\right)\right]=E\left[\sum_{i, j=g, r} \theta_{i} \widetilde{q}_{i}\left(p_{i}, p_{j}\right)\right]
\end{gathered}
$$

s.t.

$$
\begin{gathered}
G \widetilde{N} R\left(p_{i}, p_{j}, x_{i}, x_{j}\right) \geq F \\
E \widetilde{I} s\left(p_{i}, p_{j}, x_{i}, x_{j}\right) \leq E \\
\widetilde{\pi}_{g}\left(p_{i}, p_{j}, w_{i}, w_{j}, x_{i}, x_{j}\right) \geq \widetilde{L}_{g} \\
\widetilde{\pi}_{r}\left(p_{i}, p_{j}, w_{i}, w_{j}, x_{i}, x_{j}\right) \geq \widetilde{L}_{r} \\
\widetilde{\pi}_{M g}\left(p_{i}, p_{j}, w_{i}, w_{j}, x_{i}, x_{j}\right) \geq L_{M g} \\
\widetilde{\pi}_{M r}\left(p_{i}, p_{j}, w_{i}, w_{j}, x_{i}, x_{j}\right) \geq L_{M r} \\
\tilde{\pi}_{R g}\left(p_{i}, p_{j}, w_{i}, w_{j}, x_{i}, x_{j}\right) \geq L_{R g} \\
\widetilde{\pi}_{R r}\left(p_{i}, p_{j}, w_{i}, w_{j}, x_{i}, x_{j}\right) \geq L_{R r}
\end{gathered}
$$




$$
x_{g}, x_{r} \geq 0
$$

Substituting $p_{g}^{*}, p_{r}^{*}, w_{g}^{*}$, and $w_{r}^{*}$ (i.e., the best response strategies of decentralized SCs), presented in Propositions 5 and 6, into the government's model in (59)-(69), the government's decision variables $\left(x_{g}^{*}\right.$ and $\left.x_{r}^{*}\right)$ can be obtained. To this end, as in Scenario 1, the model can be converted to a single objective using the $\alpha$-cut method.

\subsection{The Proposed Methodology for Decision-Making under Ambiguity}

We now describe different steps of the developed methodology that can be used by policy makers in real policy making environments.

Step 1: Specify a key product. The key product is considered a critical product for society when its resultant pollution and environmental footprint are above a certain threshold and require special control regulations and intervention by the government.

Step 2: Identify the key rival SCs that produce and offer green and regular types of the selected product in Step 1. These SCs are leading representatives of green and regular products in the market. The design policy can be imposed on the representative SCs and applied for other SCs with some modifications and adjustments.

Step 3: Evaluate the financial dimensions of members of rival SCs and the environmental impacts of their products in society. In ambiguous environments, key parameter values can be estimated based on the highest and lowest possible values of the parameters and by using triangular or trapezoidal fuzzy numbers.

Step 4: Determine the policy type and identify the structure of the SCs. The model scenario can be determined based on the adopted policy and structure of the SCs (Figure 1).

Step 5: Determine the control parameters of the corresponding policy. The parameters such as the minimum government net income, maximum environmental impact, and lowest reserved profit for SCs and their members should be specified using local or international standards, the opinions of industry experts, and official documentation and regulations. The confidence level $(\alpha)$ should also be determined.

Step 6: Solve the fuzzy mathematical models of government. Given the selected scenario, the corresponding model of government (Sections 4.1-4.4) must be solved.

Step 7: Evaluate the obtained results and perform sensitivity analysis. Once results are obtained, sensitivity analysis on key parameters must be performed to further characterize the alternative solutions for the adopted policy. If the obtained values are not desirable, go to Step 4 and modify the control parameters or policy type.

\section{Computational Study and Discussion}

In this section, we conduct a numerical study to explain theoretical findings, examine the model properties, and compare the SC members' optimal solutions under different scenarios. Our numerical study is inspired by the Canadian government's exemplary program that promotes sustainable development goals.

Off-road vehicles and mobile equipment are known as major sources of air pollution and greenhouse gas emissions in Canada. To reduce the pollution and address the public concerns, the Canadian government has been encouraging a paradigm shift among industries and public to adopt more environmentally friendly transportation options, with the reframing of the transportation infrastructure system set as the cornerstone of their future actions (https:/ / bit.ly/3qlzvGc). The government goals include reducing national greenhouse gas emissions by $40 \%$ by 2030 and by $80 \%$ by 2050 , respectively. Table 3 depicts the relationship of the trapezoidal fuzzy variables with their linguistic expressions, which are used as fuzzy estimations in our models. 
Table 3. Relationship between triangular fuzzy variables and their linguistic expressions.

\begin{tabular}{|c|c|c|}
\hline Parameter & Linguistic Expression & Trapezoidal Fuzzy Variables \\
\hline \multirow{3}{*}{$\widetilde{a}_{g}$} & High & (10.5 111313.5$)$ \\
\hline & Medium & (9 1011 12) \\
\hline & Small & (78 88.510$)$ \\
\hline \multirow{3}{*}{$\widetilde{a}_{r}$} & High & $(101212.514)$ \\
\hline & Medium & $(8.59 .51012)$ \\
\hline & Small & $(78910)$ \\
\hline \multirow{3}{*}{$\widetilde{c}_{g}$} & High & $(66.57 .59)$ \\
\hline & Medium & $(4.566 .58)$ \\
\hline & Low & $(3567)$ \\
\hline \multirow{3}{*}{$\widetilde{c}_{r}$} & High & (4 5.56 .57$)$ \\
\hline & Medium & (3 44.254 .75$)$ \\
\hline & Low & (2.53 3.54 .25$)$ \\
\hline \multirow{3}{*}{$\widetilde{L}_{g}$} & High & $(8.51011 .512 .5)$ \\
\hline & Medium & $(89.511$ 11.5) \\
\hline & Low & $(677.58)$ \\
\hline \multirow{3}{*}{$\widetilde{L}_{r}$} & High & (8 9.510 .512$)$ \\
\hline & Medium & $(788.59)$ \\
\hline & Low & $(66.578)$ \\
\hline \multirow{3}{*}{$\widetilde{d}$} & Very sensitive & $\left(\begin{array}{llllll}0 & 0.6 & 0.8 & 0.9\end{array}\right)$ \\
\hline & Sensitive & $\left(\begin{array}{llll}0.4 & 0.48 & 0.57 & 0.7\end{array}\right)$ \\
\hline & Not very sensitive & (0.08 0.110 .260 .35$)$ \\
\hline \multirow{3}{*}{$\widetilde{L}_{M g}$} & High & $(7.58 .5910)$ \\
\hline & Medium & $(77.58 .59)$ \\
\hline & Low & (6.5 7.25 7.5 8.5) \\
\hline \multirow{3}{*}{$\widetilde{L}_{M r}$} & High & (7 88.510$)$ \\
\hline & Medium & (7.5899.5) \\
\hline & Low & $(6.577 .58)$ \\
\hline \multirow{3}{*}{$\widetilde{L}_{R g}$} & High & (1 1.2522 .5$)$ \\
\hline & Medium & (1 1 1.52 .53$)$ \\
\hline & Low & (1 1.251 .52$)$ \\
\hline \multirow{3}{*}{$\widetilde{L}_{R r}$} & High & $(2.5344 .5)$ \\
\hline & Medium & $(22.53 .54)$ \\
\hline & Low & $(1.522 .53)$ \\
\hline
\end{tabular}

The following fuzzy linguistic variables are considered to test the fuzzy parameters: green market scale: high; regular market scale: medium; green manufacturing cost: high; regular manufacturing cost: low; GSC reservation profit: low; RSC reservation profit: high; sustainability coefficient: not very sensitive; reservation profit of manufacturers and retailers in each SC: medium.

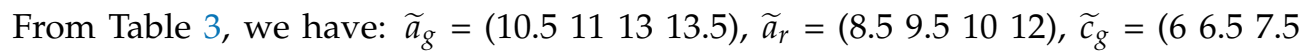

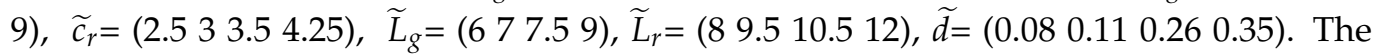
values of the nonfuzzy input parameters are: $E=12, F=150, \theta_{g}=1, \theta_{r}=2, Q_{g}=40, Q_{r}=11$, and $\omega_{k 1}=\omega_{k 2}=\omega_{k 3}=\omega_{k 4}=\omega_{k 5}=\omega_{k 6}=0.167$. Note the expected value of the fuzzy numbers were adopted from Jiménez et al. [51].

The impact of $\alpha$-cuts on the expected profit of SCs in different scenarios is illustrated in Figure 6a-d. Figure 6a shows the impact of $\alpha$-cut on the value of the decision variables. 


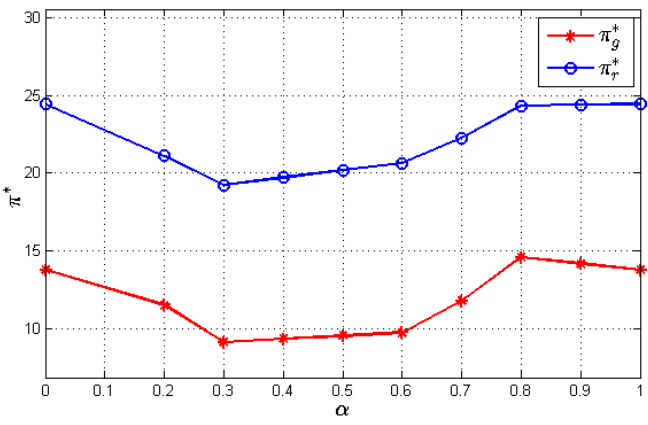

(a) Scenario 1

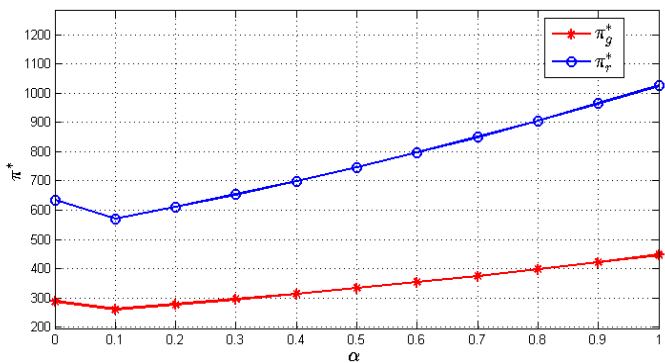

(c) Scenario 3

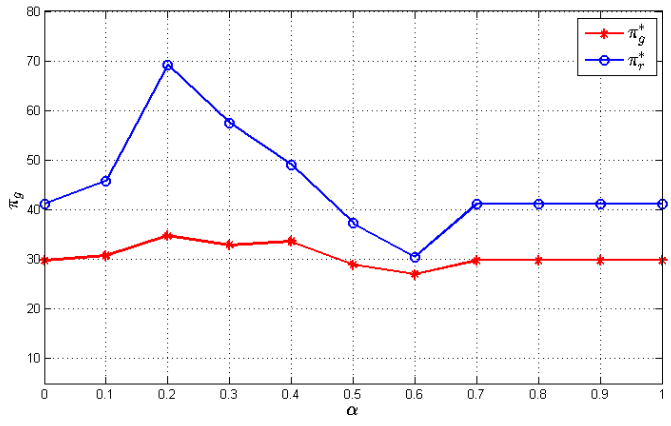

(b) Scenario 2

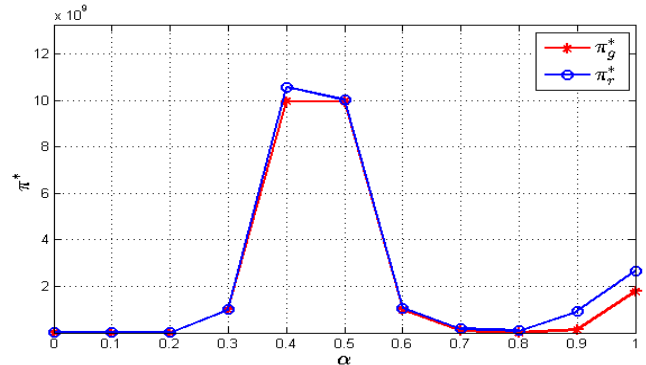

(d) Scenario 4

Figure 6. Impact of $\alpha$-cuts on the expected profit of SCs in different scenarios.

Comparing the two centralized and decentralized structures Figure $6 \mathrm{~b}$,d illustrate more benefit obtained by decentralized structures than a centralized one under the same conditions. In addition, in the decentralized structure, when the government's policies are based on production quantity, the expected total benefit for GSC and RSC is approximately similar.

Figure 7a,b summarize the result of sensitivity analysis that was conducted based on the changes in GNR and EIs under different $\alpha$-cuts. The impacts of $F$ and $E$ on the government's objectives are illustrated in Figure 8a,b.

Our results reveal that, by choosing an appropriate level of confidence ( $\alpha$-cut), managers can make a balance between reducing information ambiguity and their SC costs under different strategies (Figure 6 As reducing information ambiguity requires extra effort and carries additional costs, the preferred strategy is highly dependent on the manager's optimism and pessimism in decision-making $(0 \leq \alpha \leq 1)$. However, considering fuzzy numbers to represent information ambiguity leads to a more flexible solution space, and hence, does not necessarily increase the model complexity and solution time, especially for smaller sized problems.

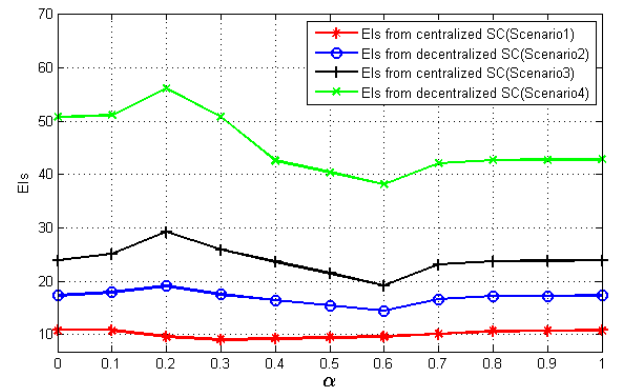

(a) The optimal EIs under different $\alpha$-cuts

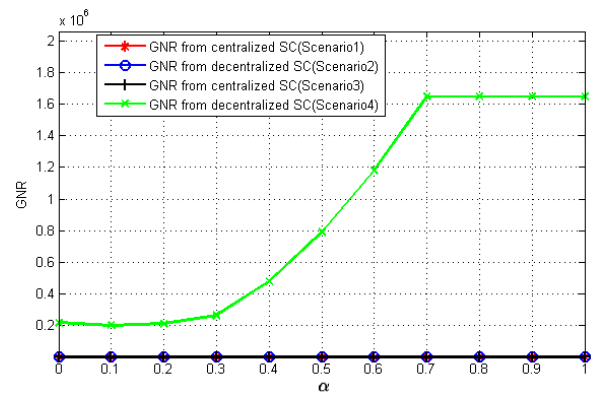

(b) The optimal GNR under different $\alpha$-cut

Figure 7. Impact of $\alpha$-cuts on the government's objectives. 


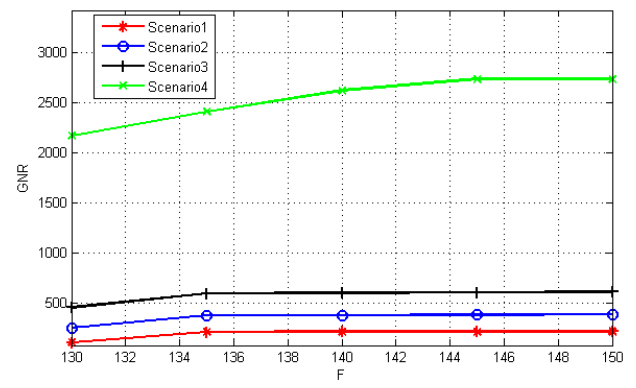

(a) The optimal GNR vs. the minimum net revenue

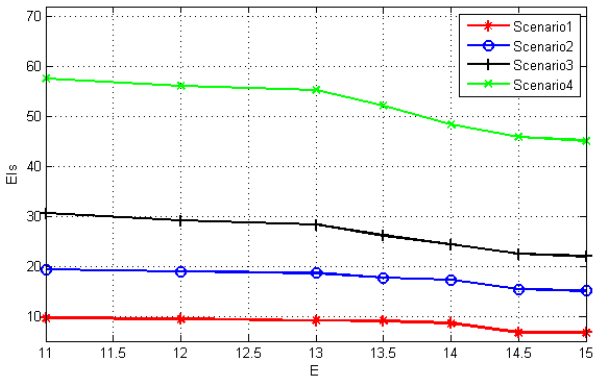

(b) The optimal EIs vs. the maximum environmental impact

Figure 8. Impact of $F$ and $E$ on the government's objectives.

\section{Conclusions}

Motivated by the growing concern regarding environmental issues, this study examined the impact of government intervention on the price and production quantity of two competing SCs (i.e., green and non-green). Considering both centralized and decentralized SC structures, a fuzzy game theoretical model was developed to capture the impact of information ambiguity on the competing game players' decisions. In this environment, we provided a step-by-step procedure for analyzing the government's policy making and examining the competing SCs' decision-making behavior. The main results of our study are as follows:

- Despite challenges, our results revealed that it is critical to incorporate information ambiguity in real-world policy making environments in which SCs' decisions are highly influenced by the fuzzy parameters.

- Regardless of the government's policy, a decentralized structure always leads to better results compared to a decentralized setting. However, taking governmental intervention into account, a green SC's manufacturer has more incentive to produce and offer green products to the shared market. Moreover, strategy intentions and the leader's attitudes toward information ambiguity strongly influence these results.

- The proposed model was optimized according to different levels of $\alpha$-cut. Hence, choosing an appropriate level of confidence $(\alpha$-cut), managers would be able to strike a balance between efforts needed to lower information ambiguity and the SC costs associated with different environmental intervention strategies.

- When government policies are focused on production quantities, a higher GNR can be obtained. Thus, when evaluating environmental policies for competing SCs, it would be more effective for governments to focus on the production quantity rather than price under the same conditions.

Like any fuzzy multi-objective optimization problem, our results were highly dependent on the decision-maker's personal beliefs. So, the results may vary in different environments depending on the decision-maker's attitude towards information ambiguity and the dynamics of environmental policy making ecosystem. Some possible directions for future research can be proposed. For instance, considering a multi-player or multi-product model could result in selecting different intervention strategies with different combinations of tariffs and subsidies. The presented model can be generalized by adding more fuzzy variables and/or employing other fuzzy programming approaches. Following the mainstream research on SC and logistics risk management [53-57], the developed models in this paper can be extended to incorporate and address both risk and ambiguity.

Author Contributions: Conceptualization, M.R., A.H., S.A., L.M.; methodology, M.R., A.H., S.A.; validation, S.A., A.H., L.M.; formal analysis, M.R., A.H.; investigation, M.R., A.H., S.A., L.M.; resources, M.R., A.H., S.A., L.M.; writing-original draft preparation, M.R., A.H., S.A., L.M.; writingreview and editing, M.R., A.H., S.A., L.M.; visualization, M.R., A.H., S.A.; supervision, S.A., A.H., 
L.M.; funding acquisition, S.A., L.M. All authors have read and agreed to the published version of the manuscript.

Funding: This research was partially funded by the Spanish Ministry of Economy and Competitiveness through the Spanish National Research Projects PGC2018-099402-B-I00. This research was also partially supported by a Social Research Platform Grant from La Trobe University.

Institutional Review Board Statement: Not applicable for studies not involving humans or animals.

Data Availability Statement: No real data is used in this study.

Acknowledgments: We sincerely thank the editors and anonymous reviewers for their constructive and insightful comments, which led to major improvements.

Conflicts of Interest: The authors declare no conflict of interest.

\section{Appendix A}

Proof of Proposition 1. $p_{g}$ and $p_{r}$ are independent variables of each chain's profit function. Hence, using Equation (2), we have:

$$
\begin{gathered}
\frac{\partial E\left[\widetilde{\pi}_{i}\left(p_{i}, p_{j}, t_{i}, t_{j}\right)\right]}{\partial p_{i}}=0 \rightarrow-2 p_{i}\left(t_{i}, t_{j}\right)+p_{j}\left(t_{i}, t_{j}\right) \int_{0}^{1} 1 / 2\left(\widetilde{d}_{\alpha}^{L}+\widetilde{d}_{\alpha}^{U}\right) \mathrm{d} \alpha-t_{i}+ \\
1 / 2 \int_{0}^{1}\left[\left(\widetilde{a}_{i \alpha}^{L}+\widetilde{a}_{i \alpha}^{U}\right)+\left(\widetilde{c}_{i \alpha}^{L}+\widetilde{c}_{i \alpha}^{U}\right)\right] \mathrm{d} \alpha+ \\
t_{j}\left[\int_{0}^{1} 1 / 2\left(\left(\widetilde{d}_{\alpha}^{L}+\widetilde{d}_{\alpha}^{U}\right) \mathrm{d} \alpha\right)\right]^{2}=0 ; i, j=g, r ; i \neq j
\end{gathered}
$$

After simplification, the optimum price of each SC can be obtained as follows:

$$
p_{i}^{*}\left(t_{i}, t_{j}\right)=A_{i}+\frac{E[\widetilde{d}]^{2}-2}{4-E[\widetilde{d}]^{2}} t_{i}+\frac{E[\widetilde{d}]}{4-E[\widetilde{d}]^{2}} t_{j} ; i, j=g, r ; i \neq j
$$

where, $A_{i}=\frac{E\left[2 \widetilde{a}_{i}+2 \widetilde{c}_{j}+\widetilde{d}_{j}+\widetilde{d}_{i}\right]}{4-E[\widetilde{d}]^{2}}$

Proof of Proposition 2. Under a decentralized structure in Scenario 2, the expected profit of SCs' members should be calculated separately. The expected profit of retailer $i$ (see Equation (24)) can be calculated as:

$$
\begin{aligned}
E\left[\widetilde { \pi } _ { R i } \left(p_{i}, p_{j}, t_{i}, t_{j},\right.\right. & \left.\left.w_{i}, w_{j}\right)\right]=E\left[\left(p_{i}-w_{i}\right) \widetilde{q}_{i}\right] \\
& =E\left[\left(p_{i}-w_{i}\right)\left(\widetilde{a}_{i}-\left(p_{i}+t_{i}\right)+\widetilde{d}\left(p_{j}+t_{j}\right)\right)\right]
\end{aligned}
$$

using $\alpha$-optimistic and $\alpha$-pessimistic value, we have:

$$
\begin{aligned}
E\left[\widetilde{\pi}_{R i}\left(p_{i}, p_{j}, t_{i}, t_{j}, \quad w_{i}, w_{j}\right)\right] \\
=\left(p_{i}-w_{i}\right) \int_{0}^{1} \frac{1}{2}\left(\left(\widetilde{a}_{i \alpha}^{L}+\widetilde{a}_{i \alpha}^{U}\right)\right) \mathrm{d} \alpha-\left(p_{i}-w_{i}\right)\left(p_{i}+t_{i}\right) \\
+\left(p_{i}-w_{i}\right)\left(p_{j}+t_{j}\right) E[\widetilde{d}]
\end{aligned}
$$


As in the proof of Proposition 1, considering $\frac{\partial E\left[\widetilde{\pi}_{R i}\left(p_{i}, p_{j}, t_{i}, t_{j}, w_{i}, w_{j}\right)\right]}{\partial p_{i}}=0$, we have:

$$
\begin{aligned}
-2 p_{i}\left(t_{i}, t_{j}, w_{i}, w_{j}\right) & +p_{j}\left(t_{i}, t_{j}, w_{i}, w_{j}\right) \int_{0}^{1} \frac{1}{2}\left(\widetilde{d}_{\alpha}^{L}+\widetilde{d}_{\alpha}^{U}\right) \mathrm{d} \alpha-t_{i} \\
& +t_{j}\left[\int_{0}^{1} \frac{1}{2}\left(\widetilde{d}_{\alpha}^{L}+\widetilde{d}_{\alpha}^{U}\right) \mathrm{d} \alpha\right]^{2}+\frac{1}{2} \int_{0}^{1}\left(\widetilde{a}_{i \alpha}^{L}+\widetilde{a}_{i \alpha}^{U}\right) \mathrm{d} \alpha+w_{i}=0
\end{aligned}
$$

As a result, the optimum retail price of each SC can be obtained as follows:

$$
p_{i}^{*}\left(t_{i}, t_{j}, w_{i}, w_{j}\right)=B_{i}+\frac{2}{4-E[\tilde{d}]^{2}}\left(w_{i}\right)+\frac{E[\tilde{d}]}{4-E[\tilde{d}]^{2}}\left(w_{j}\right)
$$

where, $B_{i}=\frac{E\left[2 \widetilde{a}_{i}+\widetilde{d}_{a_{j}}\right]-\left(E[\tilde{d}]^{2}-2\right) t_{i}+E[\tilde{d}] t_{j}}{4-E[\tilde{d}]^{2}} ; i, j=g, r ; i \neq j$

Proof of Proposition 3. Like the proof provided for Proposition 2, the expected profit of the SCs' members should be calculated separately. The expected profit of manufacturer $i$ (see Equation (25)) can be calculated as:

$$
\begin{aligned}
E\left[\widetilde { \pi } _ { M i } \left(p_{i}, p_{j}, t_{i}, t_{j}, \quad\right.\right. & \left.\left.w_{i}, w_{j}\right)\right] \\
& =E\left[\left(w_{i}-\widetilde{c}_{i}\right) \widetilde{q}_{i}\right] \\
& =E\left[\left(w_{i}-\widetilde{c}_{i}\right)\left(\widetilde{a}_{i}-\left(p_{i}^{*}+t_{i}\right)+\widetilde{d}\left(p_{j}^{*}+t_{j}\right)\right)\right] \\
& =E\left[\widetilde{a}_{i}\right]-w_{i}\left(p_{i}^{*}+t_{i}\right)+w_{i}\left(p_{j}^{*}+t_{j}\right) E[\widetilde{d}]-E\left[\widetilde{c}_{i} \widetilde{a}_{i}\right] \\
& +\left(p_{i}^{*}+t_{i}\right) E\left[\widetilde{c}_{i}\right]-\left(p_{j}^{*}+t_{j}\right) E\left[\widetilde{c}_{i} \widetilde{d}\right] \\
& =w_{i} \int_{0}^{1} \frac{1}{2}\left(\widetilde{a}_{i \alpha}^{L}+\widetilde{a}_{i \alpha}^{U}\right) \mathrm{d} \alpha-w_{i}\left(p_{i}^{*}+t_{i}\right) \\
& +w_{i}\left(p_{j}^{*}+t_{j}\right) \int_{0}^{1} \frac{1}{2}\left(\widetilde{d}_{\alpha}^{L}+\widetilde{d}_{\alpha}^{U}\right) \mathrm{d} \alpha \\
& +\left(p_{i}^{*}+t_{i}\right) \frac{1}{2} \int_{0}^{1}\left[\left(\widetilde{c}_{i \alpha}^{L}+\widetilde{c}_{i \alpha}^{U}\right)\right] \mathrm{d} \alpha \\
& -\left(p_{j}^{*}+t_{j}\right) \int_{0}^{1} \frac{1}{2}\left(\widetilde{d}_{\alpha}^{L} \widetilde{c}_{i \alpha}^{U}+\widetilde{d}_{\alpha}^{U} \widetilde{c}_{i \alpha}^{L}\right) \mathrm{d} \alpha
\end{aligned}
$$

After substituting the values obtained for $\left(p_{i}^{*}\left(t_{i}, t_{j}, w_{i}, w_{j}\right)\right)$ in the proof of Proposition 2 into Equation (6), we have:

$$
\begin{aligned}
E\left[\widetilde{\pi}_{M i}\left(p_{i}, p_{j}, t_{i}, t_{j}, \quad w_{i}, w_{j}\right)\right] & =E\left[( w _ { i } - \widetilde { c } _ { i } ) \left(\widetilde{a}_{i}\right.\right. \\
& -\left(B_{i}+\frac{2}{4-E[\widetilde{d}]^{2}}\left(w_{i}\right)+\frac{E[\widetilde{d}]}{4-E[\widetilde{d}]^{2}}\left(w_{j}\right)+t_{i}\right) \\
& \left.\left.+\widetilde{d}\left(B_{j}+\frac{2}{4-E[\widetilde{d}]^{2}}\left(w_{j}\right)+\frac{E[\tilde{d}]}{4-E[\widetilde{d}]^{2}}\left(w_{i}\right)+t_{j}\right)\right)\right]
\end{aligned}
$$

Therefore, from $\frac{\partial E\left[\widetilde{\pi}_{M i}\left(t_{i}, t_{j}, w_{i}, w_{j}\right)\right]}{\partial p_{i}}=0$, the manufacturer's optimal wholesale price can be obtained as follows: $w_{i}^{*}\left(t_{i}, t_{j}\right)=C_{i}+C_{1} t_{i}+C_{2} t_{j}$

where

$$
\begin{gathered}
C_{i}=\left(E\left[\left(2 \widetilde{d}^{4}-8 \widetilde{d}^{2}+8\right) \widetilde{c}_{i}\right]-E\left[\left(\widetilde{d}^{3}-2 \widetilde{d}\right) \widetilde{c}_{j}\right]-E\left[\left(-2 \widetilde{d}^{3}+6 \widetilde{d}\right) \widetilde{a}_{j}\right]+E\left[\left(-3 \widetilde{d}^{2}+8\right) \widetilde{a}_{i}\right]\right) / \\
\left(E\left[4 \widetilde{d}^{4}-17 \widetilde{d}^{2}+16\right]\right)
\end{gathered}
$$




$$
C_{1}=E\left[-5 \widetilde{d}^{4}-2 \widetilde{d}^{3}+25 \widetilde{d}^{2}+4 \widetilde{d}-24\right] / E\left[4 \widetilde{d}^{4}-17 \widetilde{d}^{2}+16\right]
$$

and

$$
C_{2}=\frac{E\left[2 \widetilde{d}^{5}+2 \widetilde{d}^{4}-2 \widetilde{d}^{3}+14 \widetilde{d}\right]}{E\left[4 \widetilde{d}^{4}-17 \widetilde{d}^{2}+16\right]}
$$

Proof of Proposition 4. Under the centralized SC structure, the expected profit of each SC can be derived as follows (Equations (40)-(41)):

Profit for GSC: $E\left[\widetilde{\pi}_{g}\left(p_{g}, p_{r}, x_{g}, x_{r}\right)\right]=E\left[\left(p_{g}-\widetilde{c}_{g}\right) \widetilde{q}_{g}\left(p_{g}, p_{r}\right)+x_{g}\left(\widetilde{q}_{g}\left(p_{g}, p_{r}\right)-Q_{g}\right)\right]$

Profit for RSC: $E\left[\tilde{\pi}_{r}\left(p_{g}, p_{r}, x_{g}, x_{r}\right)\right]=E\left[\left(p_{r}-\widetilde{c}_{r}\right) \widetilde{q}_{r}\left(p_{g}, p_{r}\right)-x_{r}\left(\widetilde{q}_{r}\left(p_{g}, p_{r}\right)-Q_{r}\right)\right]$

To find the manufacturer's optimal wholesale price, we derive each of the equations related to variables that have control. Thus, using $\frac{\partial E\left[\tilde{\pi}_{g}\left(p_{g}, p_{r}, x_{g}, x_{r}\right)\right]}{\partial p_{g}}=0$ and $\frac{\partial E\left[\tilde{\pi}_{r}\left(p_{g}, p_{r}, x_{g}, x_{r}\right)\right]}{\partial p_{r}}=$ 0 , we have:

$$
\frac{1}{2} \int_{0}^{1}\left[\left(\widetilde{c}_{g \alpha}^{L}+\widetilde{c}_{g \alpha}^{U}\right)+\left(\widetilde{a}_{g \alpha}^{L}+\widetilde{a}_{g \alpha}^{U}\right)\right] \mathrm{d} \alpha-2 p_{g}\left(x_{g}, x_{r}\right)-x_{g}+p_{r}\left(x_{g}, x_{r}\right) \int_{0}^{1} \frac{1}{2}\left(\widetilde{d}_{\alpha}^{L}+\widetilde{d}_{\alpha}^{U}\right) \mathrm{d} \alpha=0
$$

and

$$
\frac{1}{2} \int_{0}^{1}\left[\left(\widetilde{c}_{r \alpha}^{L}+\widetilde{c}_{r \alpha}^{U}\right)+\left(\widetilde{a}_{r \alpha}^{L}+\widetilde{a}_{r \alpha}^{U}\right)\right] \mathrm{d} \alpha-2 p_{r}\left(x_{g}, x_{r}\right)+x_{r}+p_{g}\left(x_{g}, x_{r}\right) \int_{0}^{1} \frac{1}{2}\left(\widetilde{d}_{\alpha}^{L}+\widetilde{d}_{\alpha}^{U}\right) \mathrm{d} \alpha=0
$$

After some simplifications, the optimal wholesale price can be found as follows:

$$
\begin{aligned}
& p_{g}^{*}\left(x_{i}, x_{j}\right)=D_{1 g}-\frac{2}{4-E[\tilde{d}]^{2}} x_{g}+\frac{E[\tilde{d}]}{4-E[\tilde{d}]^{2}} x_{r} \\
& p_{r}^{*}\left(x_{i}, x_{j}\right)=D_{2 r}-\frac{E[\tilde{d}]}{4-E[\tilde{d}]^{2}} x_{g}+\frac{2}{4-E[\tilde{d}]^{2}} x_{r}
\end{aligned}
$$

where, $D_{1 g}=\frac{2 E\left[\widetilde{a}_{g}+\widetilde{c}_{g}\right]+E\left[\widetilde{a}_{r} \widetilde{d}+\widetilde{c}_{r} \tilde{d}\right]}{4-E[\widetilde{d}]^{2}}$ and $D_{2 r}=\frac{2 E\left[\widetilde{a}_{r}+\widetilde{c}_{r}\right]+E\left[\widetilde{a}_{g} \widetilde{d}+\widetilde{c}_{g} \widetilde{d}\right]}{4-E[\widetilde{d}]^{2}}$

Proof of Proposition 5. As in previous proofs, from Equation (53), we have:

$$
\frac{\partial E\left[\tilde{\pi}_{R i}\left(p_{i}, p_{j}, x_{i}, x_{j}, w_{i}, w_{j}\right)\right]}{\partial p_{i}}=0
$$

After simplifications, we have:

$$
E\left[\widetilde{a}_{i}\right]-2 p_{i}+p_{j} E[\widetilde{d}]+w_{i}=0
$$

Therefore:

$$
p_{i}^{*}\left(x_{i}, x_{j}, w_{i}, w_{j}\right)=H_{i}+\frac{2}{1-E\left[4 \widetilde{d}^{2}\right]} w_{i}+\frac{E[\tilde{d}]}{4-E[\widetilde{d}]^{2}} w_{j}
$$

where $H_{i}=\frac{E\left[2 \widetilde{a}_{i}+\widetilde{d}_{j}\right]}{1-E\left[4 \widetilde{d}^{2}\right]}$

Proof of Proposition 6. After finding $p_{i}^{*}\left(x_{i}, x_{j}, w_{i}, w_{j}\right)$ in Proposition 5 and replacing them in Equations (54)-(55), we have two equations with variables $x_{g}$ and $x_{r}$. 
By solving those two equations, we have Equations (57)-(58) for $w_{g}^{*}\left(x_{g}, x_{r}\right)$ and $w_{r}^{*}\left(x_{g}, x_{r}\right)$.

$$
\begin{aligned}
& w_{g}^{*}\left(x_{g}, x_{r}\right)= \\
& \frac{E\left[\widetilde{d}^{4}\right]\left(2 x_{g}+4\right)+E\left[\widetilde{d}^{3}\right]\left(2 E\left[\widetilde{a}_{r}\right]+4 E\left[\widetilde{c}_{g}\right]+E\left[\widetilde{c}_{r}\right]+4\right)+E\left[\widetilde{d}^{2}\right]\left(3 E\left[\widetilde{a}_{g}\right]+4 E\left[\widetilde{c}_{g}\right]-8 x_{g}-8\right)}{4 E\left[\widetilde{d}^{4}\right]-17 E\left[\widetilde{d}^{2}\right]+16} \\
& +\frac{-E[\widetilde{d}]\left(6 E\left[\widetilde{a}_{r}\right]+8 E\left[\widetilde{c}_{g}\right]+2 E\left[\widetilde{c}_{r}\right]-2 x_{r}+8\right)+8\left(x_{g}-E\left[\widetilde{a}_{g}\right]-E\left[\widetilde{c}_{g}\right]\right)}{4 E\left[\widetilde{d}^{4}\right]-17 E\left[\widetilde{d}^{2}\right]+16} \\
& \frac{2 E\left[\widetilde{d}^{4}\right] E\left[\widetilde{c}_{r}\right]+E\left[\widetilde{d}^{3}\right]\left(-3 E\left[\widetilde{a}_{r}\right]+2 E\left[\widetilde{c}_{g}\right]-8 E\left[\widetilde{c}_{r}\right]+2\right)+E\left[\widetilde{d}^{2}\right]\left(2-3 E\left[\widetilde{a}_{r}\right]+2 E\left[\widetilde{c}_{g}\right]-8 E\left[\widetilde{c}_{r}\right]+8 x_{r}\right)}{4 E\left[\widetilde{d}^{4}\right]-17 E\left[\widetilde{d}^{2}\right]+16} \\
& +\frac{E[\widetilde{d}]\left(6 E\left[\widetilde{a}_{g}\right]+2 E\left[\widetilde{c}_{g}\right]-2 x_{g}\right)+8\left(-x_{r}+E\left[\widetilde{a}_{r}\right]+E\left[\widetilde{c}_{r}\right]\right)}{4 E\left[\widetilde{d}^{4}\right]-17 E\left[\widetilde{d}^{2}\right]+16}
\end{aligned}
$$

\section{References}

1. Ninlawan, C.; Seksan, P.; Tossapol, K.; Pilada, W. The implementation of green supply chain management practices in electronics industry. In World Congress on Engineering, London, UK, July 4-6, 2012; International Association of Engineers: Hong Kong, 2010; pp. 1563-1568.

2. Asian, S.; Hafezalkotob, A.; John, J.J. Sharing economy in organic food supply chains: A pathway to sustainable development. Int. J. Prod. Econ. 2019, 218, 322-338. [CrossRef]

3. Elansky, N.F; Ponomarev, N.A.; Verevkin, Y.M. Air quality and pollutant emissions in the Moscow megacity in 2005-2014. Atmos. Environ. 2018, 175, 54-64. [CrossRef]

4. Javadi, T.; Alizadeh-Basban, N.; Asian, S.; Hafezalkotob, A. Pricing policies in a dual-channel supply chain considering flexible return and energy-saving regulations. Comput. Ind. Eng. 2019, 135, 655-674. [CrossRef]

5. Lu, D.; Asian, S.; Ertek, G.; Sevinc, M. Mind the perception gap: An integrative performance management framework for service supply chains. Int. J. Phys. Distrib. Logist. Manag. 2019, 49, 33-51. [CrossRef]

6. Lu, D.; Ding, Y.; Asian, S.; Paul, S.K. From Supply Chain Integration to Operational Performance: The Moderating Effect of Market Uncertainty. Glob. J. Flex. Syst. Manag. 2017, 19, 3-20. [CrossRef]

7. De Albuquerque, G.A.; Maciel, P.; Lima, R.M.F.; Magnani, F. Strategic and tactical evaluation of conflicting environment and business goals in green supply chains. IEEE Trans. Syst. Man Cybern. Syst. 2013, 43, 1013-1027. [CrossRef]

8. Yang, D.; Xiao, T. Pricing and green level decisions of a green supply chain with governmental interventions under fuzzy uncertainties. J. Clean. Prod. 2017, 149, 1174-1187. [CrossRef]

9. Sheu, J.-B.; Chen, Y.J. Impact of government financial intervention on competition among green supply chains. Int. J. Prod. Econ. 2012, 138, 201-213. [CrossRef]

10. Zhao, J.; Tang, W.; Zhao, R.; Wei, J. Pricing decisions for substitutable products with a common retailer in fuzzy environments. Eur. J. Oper. Res. 2012, 216, 409-419. [CrossRef]

11. Wei, J.; Zhao, J. Pricing decisions with retail competition in a fuzzy closed-loop supply chain. Expert Syst. Appl. 2011, 38, 11209-11216. [CrossRef]

12. Alkhayyal, B.A.; Gupta, S.M. The Impact of Carbon Emissions Policies on Reverse Supply Chain Network Design. Doğuş Üniv. Derg. 2018, 19, 99-111. [CrossRef]

13. Asian, S.; Wang, J; Dickson, G. Trade disruptions, behavioral biases, and social influences: Can luxury sporting goods supply chains be immunized? Transp. Res. Part E Logist. Transp. Rev. 2020, 143, 102064. [CrossRef]

14. Reza-Gharehbagh, R.; Hafezalkotob, A.; Asian, S.; Makui, A.; Zhang, A.N. Peer-to-peer financing choice of SME entrepreneurs in the re-emergence of supply chain localization. Int. Trans. Oper. Res. 2020, 27, 2534-2558. [CrossRef]

15. Wei, C.; Asian, S.; Ertek, G.; Hu, Z.-H. Location-based pricing and channel selection in a supply chain: A case study from the food retail industry. Ann. Oper. Res. 2018, 291, 959-984. [CrossRef]

16. Asian, S.; Nie, X. Coordination in Supply Chains With Uncertain Demand and Disruption Risks: Existence, Analysis, and Insights. IEEE Trans. Syst. Man, Cybern. Syst. 2014, 44, 1139-1154. [CrossRef]

17. Hadi, T.; Sheikhmohammady, M.; Chaharsooghi, S.K.; Hafezalkotob, A. Competition between regular and closed-loop supply chains under financial intervention of government; a game theory approach. J. Ind. syst. eng. 2021, 13, 179-199.

18. Li, W.; Chen, J. Backward integration strategy in a retailer Stackelberg supply chain. Omega 2018, 75, 118-130. [CrossRef]

19. Chen, X.; Wang, X.; Gong, K. The Effect of Bidimensional Power Structure on Supply Chain Decisions and Performance. IEEE Trans. Syst. Man, Cybern. Syst. 2020, 50, 1095-1110. [CrossRef]

20. Wang, Y.; Lin, Y.; Chen, L.; Shi, J. A Stackelberg Game-Based Caching Incentive Scheme for Roadside Units in VANETs. Sensors 2020, 20, 6625. [CrossRef] [PubMed] 
21. Bector, C.R.; Chandra, S. Fuzzy Mathematical Programming and Fuzzy Matrix Games; Springer: Berlin, Germany, 2005.

22. Nishizaki, I.; Sakawa, M. Fuzzy and Multiobjective Games for Conflict Resolution; Springer, Physica-Verlag: Heidelberg, Germany, 2001.

23. Cunlin, L.; Qiang, Z. Nash equilibrium strategy for fuzzy non-cooperative games. Fuzzy Sets Syst. 2011, 176, 46-55. [CrossRef]

24. Oliveira, H., Jr.; Petraglia, A. Establishing nash equilibria of strategic games: A multistart fuzzy adaptive simulated annealing approach. Appl. Soft Comput. 2014, 19, 188-197. [CrossRef]

25. Gumus, A.T.; Güneri, A.F. A multi-echelon inventory management framework for stochastic and fuzzy supply chains. Expert Syst. Appl. 2009, 36, 5565-5575. [CrossRef]

26. Zhao, J.; Wang, L. Pricing and retail service decisions in fuzzy uncertainty environments. Appl. Math. Comput. 2015, 250, 580-592. [CrossRef]

27. Feng, C.; Ma, Y.; Zhou, G.; Ni, T. Stackelberg game optimization for integrated production-distribution-construction system in construction supply chain. Knowl.-Based Syst. 2018, 157, 52-67. [CrossRef]

28. Wei, J.; Zhao, J. Reverse channel decisions for a fuzzy closed-loop supply chain. Appl. Math. Model. 2013, 37, 1502-1513. [CrossRef]

29. Jamali, M.-B.; Gorji, M.-A.; Iranpoor, M. Pricing policy on a dual competitive channel for a green product under fuzzy conditions. Neural Comput. Appl. 2021,1-13.

30. Wang, Y. Pricing and Warranty Decisions of Substitutable Products for a Fuzzy Two-Echelon Supply Chain. Discret. Dyn. Nat. Soc. 2017, 2017, 1-12. [CrossRef]

31. Zhao, J.; Wei, J.; Sun, X. Coordination of fuzzy closed-loop supply chain with price dependent demand under symmetric and asymmetric information conditions. Ann. Oper. Res. 2016, 257, 469-489. [CrossRef]

32. Fallah, H.; Eskandari, H.; Pishvaee, M.S. Competitive closed-loop supply chain network design under uncertainty. J. Manuf. Syst. 2015, 37, 649-661. [CrossRef]

33. Alamdar, S.F.; Rabbani, M.; Heydari, J. Pricing, collection, and effort decisions with coordination contracts in a fuzzy, three-level closed-loop supply chain. Expert Syst. Appl. 2018, 104, 261-276. [CrossRef]

34. Tsireme, A.I.; Nikolaou, E.I.; Georgantzis, N.; Tsagarakis, K.P. The influence of environmental policy on the decisions of managers to adopt G-SCM practices. Clean Technol. Environ. Policy 2012, 14, 953-964. [CrossRef]

35. Wang, K.-L.; Zhao, B.; Ding, L.-L.; Miao, Z. Government intervention, market development, and pollution emission efficiency: Evidence from China. Sci. Total Environ. 2021, 757, 143738. [CrossRef]

36. Mahmoudi, R.; Rasti-Barzoki, M. Sustainable supply chains under government intervention with a real-world case study: An evolutionary game theoretic approach. Comput. Ind. Eng. 2018, 116, 130-143. [CrossRef]

37. Chen, H.; Dong, Z.; Li, G. Government Reward-Penalty Mechanism in Dual-Channel Closed-Loop Supply Chain. Sustainability 2020, 12, 8602. [CrossRef]

38. Jin, C.; Mei, L. Game Analysis of Multi-Strategy Between Government and Suppliers in Green Supply Chain. In Green Communications and Networks; Springer: Dordrecht, The Netherlands, 2012; pp. 185-191.

39. Hafezalkotob, A. Competition of two green and regular supply chains under environmental protection and revenue seeking policies of government. Comput. Ind. Eng. 2015, 82, 103-114. [CrossRef]

40. Hafezalkotob, A. Direct and indirect intervention schemas of government in the competition between green and non-green supply chains. J. Clean. Prod. 2018, 170, 753-772. [CrossRef]

41. Wu, C.-H. A dynamic perspective of government intervention in a competitive closed-loop supply chain. Eur. J. Oper. Res. 2021. [CrossRef]

42. Reza-Gharehbagh, R.; Hafezalkotob, A.; Makui, A.; Sayadi, M.K. Government intervention policies in competition of financial chains: A game theory approach. Kybernetes 2019, 49, 960-981. [CrossRef]

43. Wang, M.; Liu, K.; Choi, T.-M.; Yue, X. Effects of Carbon Emission Taxes on Transportation Mode Selections and Social Welfare. IEEE Trans. Syst. Man Cybern. Syst. 2015, 45, 1413-1423. [CrossRef]

44. Hafezalkotob, A.; Borhani, S.; Zamani, S. Development of a Cournot-oligopoly model for competition of multi-product supply chains under government supervision. Sci. Iran. 2017, 24, 1519-1532. [CrossRef]

45. Moradinasab, N.; Amin-Naseri, M.R.; Behbahani, T.J.; Jafarzadeh, H. Competition and cooperation between supply chains in multi-objective petroleum green supply chain: A game theoretic approach. J. Clean. Prod. 2018, 170, 818-841. [CrossRef]

46. Hafezalkotob, A. Competition, cooperation, and coopetition of green supply chains under regulations on energy saving levels. Transp. Res. Part E Logist. Transp. Rev. 2017, 97, 228-250. [CrossRef]

47. Hafezalkotob, A. Modelling intervention policies of government in price-energy saving competition of green supply chains. Comput. Ind. Eng. 2018, 119, 247-261. [CrossRef]

48. Liu, S.; Xu, Z. Stackelberg game models between two competitive retailers in fuzzy decision environment. Fuzzy Optim. Decis. Mak. 2014, 13, 33-48. [CrossRef]

49. Hong, M. Sequential Game in Supply Chain Dominated by Manufacturers Considering Selling Effort in a Fuzzy Environment. Eng. Lett. 2018, 26, 117-127.

50. Sircar, R.; Ledvina, A.F. Dynamic Bertrand Oligopoly. SSRN Electron. J. 2010, 11-44. Available online: https:/ / papers.ssrn.com/ sol3/papers.cfm?abstract_id=1587347 (accessed on 18 January 2020). [CrossRef]

51. Giannoccaro, I.; Pontrandolfo, P. Supply chain coordination by revenue sharing contracts. Int. J. Prod. Econ. 2004, 89, 131-139. [CrossRef] 
52. Jiménez, M.; Arenas, M.; Bilbao, A.; Rodriguez, M.V. Linear programming with fuzzy parameters: An interactive method resolution. Eur. J. Oper. Res. 2007, 177, 1599-1609. [CrossRef]

53. Faghih-Roohi, S.; Ong, Y.S.; Asian, S.; Zhang, A.N. Dynamic conditional value-at-risk model for routing and scheduling of hazardous material transportation networks. Ann. Oper. Res. 2016, 247, 715-734. [CrossRef]

54. Yu, B.; Guo, Z.; Asian, S.; Wang, H.; Chen, G. Flight delay prediction for commercial air transport: A deep learning approach. Transp. Res. Part E Logist. Transp. Rev. 2019, 125, 203-221. [CrossRef]

55. Paul, S.K.; Asian, S.; Goh, M.; Torabi, S.A. Managing sudden transportation disruptions in supply chains under delivery delay and quantity loss. Ann. Oper. Res. 2019, 273, 783-814. [CrossRef]

56. Asian, S.; Ertek, G.; Haksoz, C.; Pakter, S.; Ulun, S. Wind turbine accidents: A data mining study. IEEE Syst. J. 2016, 11, 1567-1578. [CrossRef]

57. Somarin, A.R.; Chen, S.; Asian, S.; Wang, D.Z. A heuristic stock allocation rule for repairable service parts. Int. J. Prod. Econ. 2017, 184, 131-140. [CrossRef] 